TRANSACTIONS OF THE

AMERICAN MATHEMATICAL SOCIETY

Volume 361, Number 2, February 2009, Pages 849-887

S 0002-9947(08)04594-7

Article electronically published on August 14, 2008

\title{
COMPLETELY 1-COMPLEMENTED SUBSPACES OF SCHATTEN SPACES
}

\author{
CHRISTIAN LE MERDY, ÉRIC RICARD, AND JEAN ROYDOR
}

\begin{abstract}
We consider the Schatten spaces $S^{p}$ in the framework of operator space theory and for any $1 \leq p \neq 2<\infty$, we characterize the completely 1complemented subspaces of $S^{p}$. They turn out to be the direct sums of spaces of the form $S^{p}(H, K)$, where $H, K$ are Hilbert spaces. This result is related to some previous work of Arazy and Friedman giving a description of all 1complemented subspaces of $S^{p}$ in terms of the Cartan factors of types 1-4. We use operator space structures on these Cartan factors regarded as subspaces of appropriate noncommutative $L^{p}$-spaces. Also we show that for any $n \geq 2$, there is a triple isomorphism on some Cartan factor of type 4 and of dimension $2 n$ which is not completely isometric, and we investigate $L^{p}$-versions of such isomorphisms.
\end{abstract}

\section{INTRODUCTION}

Let $\mathcal{H}, \mathcal{K}$ be Hilbert spaces. For any $p \geq 1$, let $S^{p}(\mathcal{H}, \mathcal{K})$ be the Schatten space of all operators $x: \mathcal{H} \rightarrow \mathcal{K}$ such that $\|x\|_{p}=\left(\operatorname{tr}\left(|x|^{p}\right)\right)^{\frac{1}{p}}$ is finite. Let $X \subset S^{p}(\mathcal{H}, \mathcal{K})$ be a (closed) subspace. We say that $X$ is 1 -complemented in $S^{p}(\mathcal{H}, \mathcal{K})$ if it is the range of a contractive projection $P: S^{p}(\mathcal{H}, \mathcal{K}) \rightarrow S^{p}(\mathcal{H}, \mathcal{K})$. In their remarkable memoirs 2, 3, Arazy and Friedman gave a complete classification of all such subspaces (for $p \neq 2$ ), in terms of Cartan factors of types 1-4.

In this paper we consider Schatten spaces and their complemented subspaces in the framework of operator spaces and completely bounded maps. Following Pisier's work [17, we regard $S^{p}(\mathcal{H}, \mathcal{K})$ as an operator space and we give a complete description of the completely 1-complemented subspaces of $S^{p}(\mathcal{H}, \mathcal{K})$, that is, spaces $X \subset S^{p}(\mathcal{H}, \mathcal{K})$ which are the range of a completely contractive projection of $S^{p}(\mathcal{H}, \mathcal{K})$.

The statement of our main result, Theorem 1.1 below, requires some tensor product definitions and some notation. For any Hilbert spaces $H, H^{\prime}, K, K^{\prime}$, we will consider the natural embedding

$$
S^{p}\left(H^{\prime}, K^{\prime}\right) \otimes S^{p}(H, K) \subset S^{p}\left(H^{\prime} \stackrel{2}{\otimes} H, K^{\prime} \stackrel{2}{\otimes} K\right),
$$

where $\stackrel{2}{\otimes}$ denotes the Hilbertian tensor product. Thus for any subspace $Z \subset$

Received by the editors March 27, 2007.

2000 Mathematics Subject Classification. Primary 46L07, 46L89, 17C65.

(C)2008 American Mathematical Society 
$S^{p}\left(H^{\prime}, K^{\prime}\right)$ and any $a \in S^{p}(H, K)$, we will regard

$$
Z \otimes a:=\{z \otimes a: z \in Z\}
$$

as a subspace of $S^{p}\left(H^{\prime} \stackrel{2}{\otimes} H, K^{\prime} \stackrel{2}{\otimes} K\right)$.

If $I, J$ are two index sets, we set $S_{I, J}^{p}=S^{p}\left(\ell_{J}^{2}, \ell_{I}^{2}\right)$ and we write $S_{I}^{p}=S_{I, I}^{p}$. With this notation, $S_{I, J}^{p} \otimes S^{p}(H, K) \subset S^{p}\left(\ell_{J}^{2}(H), \ell_{I}^{2}(K)\right)$, where $\ell_{J}^{2}(H)=\ell_{J}^{2} \stackrel{2}{\otimes} H$ is the 2-direct sum of $J$ copies of $H$.

Next we recall that if $\left(H_{\alpha}\right)_{\alpha}$ and $\left(K_{\alpha}\right)_{\alpha}$ are two families of Hilbert spaces, then we have a natural isometric embedding

$$
\underset{\alpha}{p} S^{p}\left(H_{\alpha}, K_{\alpha}\right) \subset S^{p}\left(\stackrel{2}{\oplus} H_{\alpha}, \underset{\alpha}{\stackrel{2}{\oplus}} K_{\alpha}\right),
$$

where $\stackrel{p}{\oplus} S^{p}\left(H_{\alpha}, K_{\alpha}\right)$ denotes the $p$-direct sum of the $S^{p}\left(H_{\alpha}, K_{\alpha}\right)$ 's and $\underset{\alpha}{\oplus} H_{\alpha}$ denotes the 2-direct sum of the $H_{\alpha}$ 's. This is obtained by identifying any $\left(x_{\alpha}\right)_{\alpha}$ in $\underset{\alpha}{\oplus} S^{p}\left(H_{\alpha}, K_{\alpha}\right)$ with the 'diagonal' operator $\underset{\alpha}{\stackrel{2}{\oplus}} H_{\alpha} \rightarrow \underset{\alpha}{\stackrel{2}{\oplus}} K_{\alpha}$ taking any $\left(\xi_{\alpha}\right)_{\alpha}$ to $\left(x_{\alpha}^{\alpha}\left(\xi_{\alpha}\right)\right)_{\alpha}$.

Theorem 1.1. Let $\mathcal{H}, \mathcal{K}$ be Hilbert spaces, let $1 \leq p \neq 2<\infty$ and let $X \subset S^{p}(\mathcal{H}, \mathcal{K})$ be a subspace. The following are equivalent.

(i) $X$ is completely 1-complemented in $S^{p}(\mathcal{H}, \mathcal{K})$.

(ii) $X$ is [2]-1-complemented in $S^{p}(\mathcal{H}, \mathcal{K})$.

(iii) There exist, for some set $A$, two families of indices $\left(I_{\alpha}\right)_{\alpha \in A}$ and $\left(J_{\alpha}\right)_{\alpha \in A}$, a family $\left(H_{\alpha}\right)_{\alpha \in A}$ of Hilbert spaces, as well as operators $a_{\alpha} \in S^{p}\left(H_{\alpha}\right)$, and two linear isometries

$$
U: \underset{\alpha \in A}{\oplus} \ell_{J_{\alpha}}^{2}\left(H_{\alpha}\right) \longrightarrow \mathcal{H} \quad \text { and } \quad V: \underset{\alpha \in A}{\stackrel{9}{\oplus}} \ell_{I_{\alpha}}^{2}\left(H_{\alpha}\right) \longrightarrow \mathcal{K}
$$

such that

$$
X=V\left(\stackrel{\oplus}{\alpha}_{I_{\alpha}, J_{\alpha}}^{p} \otimes a_{\alpha}\right) U^{*}
$$

(iv) There exist, for some set $A$, two families of indices $\left(I_{\alpha}\right)_{\alpha \in A}$ and $\left(J_{\alpha}\right)_{\alpha \in A}$ such that $X$ is completely isometric to the p-direct sum $\underset{\alpha}{\oplus_{\alpha}} S_{I_{\alpha}, J_{\alpha}}^{p}$.

See Definition 2.1 below for the meaning of (ii). In the above statement, the main implication is (i) $\Rightarrow$ (iii). The starting point of its proof is the Arazy-Friedman work [2, 3] giving a list of all 1-complemented subspaces of $S^{p}(\mathcal{H}, \mathcal{K})$. In Section 2 , we give some background on this classification and some preliminary results, as well as a brief account on the matricial structure of Schatten spaces and completely bounded maps on their subspaces. The strategy to prove (ii) $\Rightarrow$ (iii) consists in taking any 1-complemented $X \subset S^{p}(\mathcal{H}, \mathcal{K})$ from the Arazy-Friedman list, to exhibit a canonical contractive projection onto $X$, and to determine whether that projection is completely contractive (or [2]-1-contractive). This is mostly achieved in Sections $3-5$. Theorem 1.1 is eventually proved in Section 6 .

Let $n \geq 1$ be an integer, let $\mathcal{C}_{2 n}$ be the Clifford algebra generated by a collection $\left(\omega_{1}, \ldots, \omega_{2 n}\right)$ of Fermions, and let $F_{n} \subset \mathcal{C}_{2 n}$ be the linear span of $\left\{1, \omega_{1}, \ldots, \omega_{2 n}\right.$, $\left.\omega_{1} \cdots \omega_{2 n}\right\}$. Then let $\tau: F_{n} \rightarrow F_{n}$ be the linear mapping such that $\tau\left(\omega_{1} \cdots \omega_{2 n}\right)=$ $-\omega_{1} \cdots \omega_{2 n}$ and $\tau$ is the identity on the linear span of $\left\{1, \omega_{1}, \ldots, \omega_{2 n}\right\}$. The space $F_{n}$ is a Cartan factor of type 4 and $\tau$ is a triple isomorphism. This 'transpose map' 
plays a key role in the study of 1-complemented subspaces of $S^{p}(\mathcal{H}, \mathcal{K})$ (see Section $5)$. In Section 7, we investigate further properties of $\tau$ in the framework of operator space theory. First we show that $\|\tau\|_{c b}=(n+1) / n$. Then let $F_{n}^{p} \subset L^{p}\left(\mathcal{C}_{2 n}\right)$ be the space $F_{n}$ regarded as a subspace of the noncommutative $L^{p}$-space associated to $\mathcal{C}_{2 n}$. We determine when $\tau: F_{n}^{p} \rightarrow F_{n}^{p}$ is completely contractive (it depends on $n$ and $p$ ), and we give applications and complements.

We refer the reader to [11, 12, 13, for some work on contractive and completely contractive projections on some Cartan factors, which is somehow related to the present paper. We also mention the Ng-Ozawa paper [14 for a description of the completely 1-complemented subspaces of noncommutative $L^{1}$-spaces.

\section{BACKGROUND ON COMPLETE BOUNDEDNESS AND 1-COMPLEMENTED SUBSPACES}

We start with some preliminary facts concerning completely bounded maps on Schatten spaces and their subspaces. Let $1 \leq p<\infty$, let $\mathcal{H}, \mathcal{H}^{\prime}, \mathcal{K}, \mathcal{K}^{\prime}$ be Hilbert spaces and consider subspaces $X \subset S^{p}(\mathcal{H}, \mathcal{K})$ and $Y \subset S^{p}\left(\mathcal{H}^{\prime}, \mathcal{K}^{\prime}\right)$. For any index set $I$, we let

$$
S_{I}^{p} \stackrel{p}{\otimes} X \subset S^{p}\left(\ell_{I}^{2}(\mathcal{H}), \ell_{I}^{2}(\mathcal{K})\right)
$$

denote the completion of $S_{I}^{p} \otimes X$ induced by the embedding of $S_{I}^{p} \otimes S^{p}(\mathcal{H}, \mathcal{K})$ into the space $S^{p}\left(\ell_{I}^{2}(\mathcal{H}), \ell_{I}^{2}(\mathcal{K})\right)$.

Note that for any integer $n \geq 1, S_{n}^{p} \otimes X$ coincides with the space of all $n \times n$ matrices with entries in $X$. Let $u: X \rightarrow Y$ be a bounded linear map. We set

$$
\|u\|_{n}=\left\|I_{S_{n}^{p}} \otimes u: S_{n}^{p} \stackrel{p}{\otimes} X \longrightarrow S_{n}^{p} \stackrel{p}{\otimes} Y\right\|
$$

for any $n \geq 1$, and we say that $u$ is $[n]$-contractive if $\|u\|_{n} \leq 1$. This is equivalent to

$$
\left\|\left[u\left(x_{i j}\right)\right]\right\|_{S^{p}\left(\ell_{n}^{2}\left(\mathcal{H}^{\prime}\right), \ell_{n}^{2}\left(\mathcal{K}^{\prime}\right)\right)} \leq\left\|\left[x_{i j}\right]\right\|_{S^{p}\left(\ell_{n}^{2}(\mathcal{H}), \ell_{n}^{2}(\mathcal{K})\right)}, \quad x_{i j} \in X, 1 \leq i, j \leq n .
$$

Next we set

$$
\|u\|_{c b}=\sup _{n \geq 1}\|u\|_{n} .
$$

By definition, $u$ is completely bounded if $\|u\|_{c b}<\infty$, and we say that $u$ is a complete contraction (or is completely contractive) if $\|u\|_{c b} \leq 1$. Also we say that $u$ is a complete isometry if $I_{S_{n}^{p}} \otimes u$ is an isometry for any $n \geq 1$.

The above definitions come from Pisier's fundamental work [17, and we wish to point out that they are consistent with the usual terminology of operator space theory. Indeed, assume that Schatten spaces are equipped with their 'natural' operator space structure introduced in Pisier's memoir. Then equip any subspace of a Schatten space with the inherited structure. With these conventions it is easy to check that the spaces $S_{n}^{p} \stackrel{p}{\otimes} X$ and $S_{n}^{p} \stackrel{p}{\otimes} Y$ coincide with the operator space valued Schatten spaces $S_{n}^{p}[X]$ and $S_{n}^{p}[Y]$ from [17, Chapter 1]. Hence it follows from [17, Lem. 1.7] that the definitions of \|\|$_{n}$ and \|\|$_{c b}$ given by (2.1) and (2.3) coincide with the ones obtained by regarding $X \subset S^{p}(\mathcal{H}, \mathcal{K})$ and $Y \subset S^{p}\left(\mathcal{H}^{\prime}, \mathcal{K}^{\prime}\right)$ as operator spaces. We shall not use much of operator space theory and we refer the interested reader to [18], [5] or [16] for basic definitions and background. 
Definition 2.1. Let $n \geq 1$ be an integer. We say that $X \subset S^{p}(\mathcal{H}, \mathcal{K})$ is $[n]-1$ complemented if $X$ is the range of an $[n]$-contractive projection $P: S^{p}(\mathcal{H}, \mathcal{K}) \rightarrow$ $S^{p}(\mathcal{H}, \mathcal{K})$. Then we say that $X$ is completely 1 -complemented if it is the range of a completely contractive projection $P: S^{p}(\mathcal{H}, \mathcal{K}) \rightarrow S^{p}(\mathcal{H}, \mathcal{K})$.

Note that $S^{2}(\mathcal{H}, \mathcal{K})$ is 'homogeneous'; that is, any bounded linear map $u$ : $S^{2}(\mathcal{H}, \mathcal{K}) \rightarrow S^{2}(\mathcal{H}, \mathcal{K})$ is automatically completely bounded, with $\|u\|_{c b}=\|u\|$ (see [18. Chap. 7]). Consequently, any $X \subset S^{2}(\mathcal{H}, \mathcal{K})$ is completely 1-complemented. Thus we will only focus on $1 \leq p \neq 2<\infty$ in the sequel.

We say that $X \subset S^{p}(\mathcal{H}, \mathcal{K})$ and $Y \subset S^{p}\left(\mathcal{H}^{\prime}, \mathcal{K}^{\prime}\right)$ are equivalent, and we write

$$
X \sim Y,
$$

if there exist two partial isometries $U: \mathcal{H}^{\prime} \rightarrow \mathcal{H}$ and $V: \mathcal{K}^{\prime} \rightarrow \mathcal{K}$ such that

$$
X=V Y U^{*} \quad \text { and } \quad Y=V^{*} X U .
$$

Note that if $X=V Y U^{*}$, then $Y=V^{*} X U$ if and only if $y=V^{*} V y U^{*} U$ for any $y \in Y$ if and only if the mapping $y \mapsto V y U^{*}$ is one-to-one on $Y$.

Lemma 2.2. Let $\mathcal{H}, \mathcal{H}^{\prime}, \mathcal{K}, \mathcal{K}^{\prime}$ be Hilbert spaces, and let $W_{1}: \mathcal{H} \rightarrow \mathcal{H}^{\prime}$ and $W_{2}: \mathcal{K} \rightarrow$ $\mathcal{K}^{\prime}$ be two contractions. Then the linear mapping $S^{p}\left(\mathcal{H}^{\prime}, \mathcal{K}^{\prime}\right) \rightarrow S^{p}(\mathcal{H}, \mathcal{K})$ taking any $z \in S^{p}\left(\mathcal{H}^{\prime}, \mathcal{K}^{\prime}\right)$ to $W_{2}^{*} z W_{1}$ is a complete contraction.

Proof. This is clear using (2.2).

Lemma 2.3. Assume that $X \subset S^{p}(\mathcal{H}, \mathcal{K})$ and $Y \subset S^{p}\left(\mathcal{H}^{\prime}, \mathcal{K}^{\prime}\right)$ are equivalent. Then $X$ and $Y$ are completely isometric and for any $n \geq 1, X$ is $[n]$-1-complemented in $S^{p}(\mathcal{H}, \mathcal{K})$ if and only if $Y$ is $[n]-1$-complemented in $S^{p}\left(\mathcal{H}^{\prime}, \mathcal{K}^{\prime}\right)$. Also, $X$ is completely 1-complemented in $S^{p}(\mathcal{H}, \mathcal{K})$ if and only if $Y$ is completely 1-complemented in $S^{p}\left(\mathcal{H}^{\prime}, \mathcal{K}^{\prime}\right)$.

Proof. Lemma 2.2 ensures that $y \mapsto V y U^{*}$ is a complete isometry from $Y$ onto $X$. Now suppose that $P: S^{p}(\mathcal{H}, \mathcal{K}) \rightarrow S^{p}(\mathcal{H}, \mathcal{K})$ is a contractive projection whose range is equal to $X$, and that $X$ and $Y$ satisfy (2.4). Then the mapping $Q: S^{p}\left(\mathcal{H}^{\prime}, \mathcal{K}^{\prime}\right) \rightarrow$ $S^{p}\left(\mathcal{H}^{\prime}, \mathcal{K}^{\prime}\right)$ defined by

$$
Q(z)=V^{*} P\left(V z U^{*}\right) U, \quad z \in S^{p}\left(\mathcal{H}^{\prime}, \mathcal{K}^{\prime}\right),
$$

is a contractive projection whose range is equal to $Y$. Moreover it follows from Lemma 2.2 that $\|Q\|_{n} \leq\|P\|_{n}$ for any integer $n \geq 1$. This implies the second part of the statement.

Remark 2.4. Although it is not apparent in the notation, the property $X \sim Y$ depends on the embeddings $X \subset S^{p}(\mathcal{H}, \mathcal{K})$ and $Y \subset S^{p}\left(\mathcal{H}^{\prime}, \mathcal{K}^{\prime}\right)$, and not only on the operator space structures of $X$ and $Y$. Namely, $X$ and $Y$ may be completely isometric without being equivalent. This subtlety should not lead to any confusion, since the embeddings considered for various spaces studied below will be clear from the context. Note also that if we have Hilbert spaces $H \subset \mathcal{H}$ and $K \subset \mathcal{K}$, then $S^{p}(H, K)$ regarded as a subspace of $S^{p}(\mathcal{H}, \mathcal{K})$ is equivalent to $S^{p}(H, K)$ regarded as a subspace of itself.

In the second part of this section, we review the classification of 1-complemented subspaces of $S^{p}(\mathcal{H}, \mathcal{K})$ obtained by Arazy-Friedman [2, 3]. We fix some $1 \leq p \neq$ $2<\infty$ throughout. 
Let $X_{1}, X_{2} \subset S^{p}(\mathcal{H}, \mathcal{K})$ be two subspaces. We say that $X_{1}$ and $X_{2}$ are orthogonal if

$$
x_{1}^{*} x_{2}=0 \quad \text { and } \quad x_{1} x_{2}^{*}=0, \quad x_{1} \in X_{1}, x_{2} \in X_{2} .
$$

As observed in [3. p. 18], this is equivalent to the identity

$$
\left\|x_{1}+x_{2}\right\|^{p}=\left\|x_{1}\right\|^{p}+\left\|x_{2}\right\|^{p}, \quad x_{1} \in X_{1}, x_{2} \in X_{2} .
$$

Also it is easy to check (left to the reader) that this is equivalent to the existence of orthogonal decompositions $\mathcal{H}=H_{1} \stackrel{2}{\oplus} H_{2}$ and $\mathcal{K}=K_{1} \stackrel{2}{\oplus} K_{2}$ such that $X_{i} \subset$ $S^{p}\left(H_{i}, K_{i}\right)$ for $i=1,2$. Consequently, if $\left(X_{\alpha}\right)_{\alpha}$ is a family of pairwise orthogonal subspaces of $S^{p}(\mathcal{H}, \mathcal{K})$, then the closed subspace of $S^{p}(\mathcal{H}, \mathcal{K})$ generated by the $X_{\alpha}$ 's is equal to their $p$-direct sum $\underset{\alpha}{p} X_{\alpha}$. Furthermore we have

$$
S_{n}^{p} \stackrel{p}{\otimes}\left(\underset{\alpha}{\oplus} X_{\alpha}\right)=\underset{\alpha}{\oplus}\left(S_{n}^{p} \stackrel{p}{\otimes} X_{\alpha}\right)
$$

for any $n \geq 1$.

We say that $X \subset S^{p}(\mathcal{H}, \mathcal{K})$ is indecomposable if it cannot be written as the direct sum of two nontrivial orthogonal subspaces. According to [3, Prop. 2.2], any subspace $X$ of $S^{p}(\mathcal{H}, \mathcal{K})$ is equal to a direct sum $\underset{\alpha}{\stackrel{p}{ }} X_{\alpha}$ of pairwise orthogonal indecomposable subspaces. For that reason we will concentrate on indecomposable subspaces in the rest of this section and in the next three sections. We note that if $X$ and $Y$ are two subspaces of some $S^{p}$-spaces, and if $X$ and $Y$ are isometric, then $X$ is indecomposable if and only if $Y$ is indecomposable. Indeed, this follows from (2.5).

For any two index sets $I$ and $J$, we regard elements of $S_{I, J}^{p}$ as scalar matrices $\left[t_{i j}\right]_{i \in I, j \in J}$ in the usual way. Then we let $\sigma: S_{I, J}^{p} \rightarrow S_{J, I}^{p}$ be the transpose map, defined by

$$
\sigma\left(\left[t_{i j}\right]\right)=\left[t_{j i}\right] .
$$

This is an isometry. In the case $J=I$, we let

$$
\mathcal{S}_{I}^{p}=\left\{w \in S_{I}^{p}: \sigma(w)=w\right\} \quad \text { and } \quad \mathcal{A}_{I}^{p}=\left\{w \in S_{I}^{p}: \sigma(w)=-w\right\}
$$

be the spaces of symmetric and anti-symmetric matrices, respectively.

It is clear that $\mathcal{S}_{I}^{p}$ and $\mathcal{A}_{I}^{p}$ are 1-complemented subspaces of $S_{I}^{p}$. Indeed,

$$
P_{s}=\frac{1}{2}(I d+\sigma) \quad \text { and } \quad P_{a}=\frac{1}{2}(I d-\sigma)
$$

are contractive projections whose ranges are equal to $\mathcal{S}_{I}^{p}$ and $\mathcal{A}_{I}^{p}$ respectively. Likewise, for any operator $a \in S^{p}(H)$ in some $S^{p}$-space, the two spaces $\mathcal{S}_{I}^{p} \otimes a$ and $\mathcal{A}_{I}^{p} \otimes a$ are 1-complemented subspaces of $S^{p}\left(\ell_{I}^{2}(H)\right)$.

Definition 2.5. We say that $X \subset S^{p}(\mathcal{H}, \mathcal{K})$ is a space of symmetric matrices (resp. of anti-symmetric matrices) if it is equivalent to a space of the form $\mathcal{S}_{I}^{p} \otimes a$ (resp. $\left.\mathcal{A}_{I}^{p} \otimes a\right)$, where $I$ is an index set and $a \in S^{p}(H)$.

Let $a_{1} \in S^{p}\left(H_{1}\right)$ and $a_{2} \in S^{p}\left(H_{2}\right)$ be two operators, and consider the spaces $Y_{1}=S_{I, J}^{p} \otimes a_{1} \subset S^{p}\left(\ell_{J}^{2}\left(H_{1}\right), \ell_{I}^{2}\left(H_{1}\right)\right)$ and $Y_{2}=S_{J, I}^{p} \otimes a_{2} \subset S^{p}\left(\ell_{I}^{2}\left(H_{2}\right), \ell_{J}^{2}\left(H_{2}\right)\right)$,

as well as

$$
Y=\left\{\left(w \otimes a_{1}, \sigma(w) \otimes a_{2}\right): w \in S_{I, J}^{p}\right\} \subset Y_{1} \stackrel{p}{\oplus} Y_{2}
$$


In this definition we assume that $\left(a_{1}, a_{2}\right) \neq(0,0)$, excluding the trivial case $Y=$ $\{0\}$. However $a_{1}$ or $a_{2}$ can be equal to 0 .

The space $Y$ is 1-complemented in $Y_{1} \stackrel{p}{\oplus} Y_{2}$. To check this fact, and also for further purposes, it is convenient to use matrix notation. In the sequel, for any $z_{1} \in S_{I, J}^{p}, z_{2} \in S_{J, I}^{p}$ we identify $\left(z_{1} \otimes a_{1}, z_{2} \otimes a_{2}\right) \in Y_{1} \stackrel{p}{\oplus} Y_{2}$ with the $2 \times 2$ diagonal matrix

$$
\left[\begin{array}{cc}
z_{1} & 0 \\
0 & z_{2}
\end{array}\right]
$$

We may assume that $\left\|a_{1}\right\|_{p}^{p}+\left\|a_{2}\right\|_{p}^{p}=1$, and we let $t=\left\|a_{1}\right\|_{p}^{p}$. Then $\left\|z_{1} \otimes a_{1}\right\|=$ $\left\|z_{1}\right\|\left\|a_{1}\right\|=t^{\frac{1}{p}}\left\|z_{1}\right\|$, whereas $\left\|z_{2} \otimes a_{2}\right\|=(1-t)^{\frac{1}{p}}\left\|z_{2}\right\|$. Hence the norm on $Y_{1} \stackrel{p}{\oplus} Y_{2}$ in the above identification is given by

$$
\left\|\left[\begin{array}{cc}
z_{1} & 0 \\
0 & z_{2}
\end{array}\right]\right\|=\left(t\left\|z_{1}\right\|^{p}+(1-t)\left\|z_{2}\right\|^{p}\right)^{\frac{1}{p}} .
$$

Furthermore

$$
Y=\left\{\left[\begin{array}{cc}
w & 0 \\
0 & \sigma(w)
\end{array}\right]: w \in S_{I, J}^{p}\right\} .
$$

Let $P: Y_{1} \stackrel{p}{\oplus} Y_{2} \rightarrow Y_{1} \stackrel{p}{\oplus} Y_{2}$ be the linear mapping defined by

$$
P\left(\left[\begin{array}{cc}
z_{1} & 0 \\
0 & z_{2}
\end{array}\right]\right)=\left[\begin{array}{cc}
t z_{1}+(1-t) \sigma^{-1}\left(z_{2}\right) & 0 \\
0 & t \sigma\left(z_{1}\right)+(1-t) z_{2}
\end{array}\right] .
$$

It is plain that $P$ is a projection onto $Y$. Moreover by convexity we have

$$
\begin{aligned}
\left\|t z_{1}+(1-t) \sigma^{-1}\left(z_{2}\right)\right\|^{p} & \leq\left(t\left\|z_{1}\right\|+(1-t)\left\|\sigma^{-1}\left(z_{2}\right)\right\|\right)^{p} \\
& \leq t\left\|z_{1}\right\|^{p}+(1-t)\left\|\sigma^{-1}\left(z_{2}\right)\right\|^{p} \leq\left\|\left[\begin{array}{cc}
z_{1} & 0 \\
0 & z_{2}
\end{array}\right]\right\|^{p}
\end{aligned}
$$

for any $z_{1} \in S_{I, J}^{p}, z_{2} \in S_{J, I}^{p}$. Likewise,

$$
\left\|t \sigma\left(z_{1}\right)+(1-t) z_{2}\right\| \leq\left\|\left[\begin{array}{cc}
z_{1} & 0 \\
0 & z_{2}
\end{array}\right]\right\|
$$

which shows that the projection $P$ is contractive. This implies that $Z$ is 1-complemented in the $p$-direct sum of $S^{p}\left(\ell_{J}^{2}\left(H_{1}\right), \ell_{I}^{2}\left(H_{1}\right)\right)$ and $S^{p}\left(\ell_{I}^{2}\left(H_{2}\right), \ell_{I}^{2}\left(H_{2}\right)\right)$, and hence in the $S^{p}$-space

$$
S^{p}\left(\ell_{J}^{2}\left(H_{1}\right) \stackrel{2}{\oplus} \ell_{I}^{2}\left(H_{2}\right), \ell_{I}^{2}\left(H_{1}\right) \stackrel{2}{\oplus} \ell_{I}^{2}\left(H_{2}\right)\right) .
$$

Definition 2.6. We say that $X \subset S^{p}(\mathcal{H}, \mathcal{K})$ is a space of rectangular matrices if it is equivalent to a space $Y$ of the form (2.8).

We now turn to the construction of operator spaces acting on anti-symmetric Fock spaces. We refer the reader to [4, 20] for general information on these spaces. Let $n \geq 1$ be an integer. For any $k=0, \ldots, n$, we let $\Lambda_{n, k}$ denote the $k$-fold anti-symmetric tensor product of the Hilbert space $\ell_{n}^{2}$, equipped with the canonical inner product given by

$$
\left\langle\xi_{1} \wedge \cdots \wedge \xi_{k}, \xi_{1}^{\prime} \wedge \cdots \wedge \xi_{k}^{\prime}\right\rangle=\operatorname{det}\left[\left\langle\xi_{i}, \xi_{j}^{\prime}\right\rangle\right], \quad \xi_{i}, \quad \xi_{j}^{\prime} \in \ell_{n}^{2} .
$$


By convention, $\Lambda_{n, 0}=\mathbb{C}$. We let $\Omega$ be a particular unit element of $\Lambda_{n, 0}$, which is called the vacuum vector. Then the anti-symmetric Fock space over $\ell_{n}^{2}$ is the Hilbertian direct sum

$$
\Lambda_{n}=\underset{0 \leq k \leq n}{\stackrel{2}{\oplus}} \Lambda_{n, k} .
$$

Throughout we let $\left(e_{1}, \ldots, e_{n}\right)$ denote the canonical basis of $\ell_{n}^{2}$ and we let $\mathcal{P}_{n}$ be the set of all subsets of $\{1, \ldots, n\}$. Let $A \in \mathcal{P}_{n}$ with cardinality $|A|=k$, and let $1 \leq j_{1}<\cdots<j_{k} \leq n$ be the increasing enumeration of the elements of $A$. Then we set

$$
e_{A}=e_{j_{1}} \wedge \cdots \wedge e_{j_{k}} .
$$

By convention, $e_{\emptyset}=\Omega$. Clearly the system $\left\{e_{A}:|A|=k\right\}$ is an orthonormal basis of $\Lambda_{n, k}$. We will call it 'canonical' in the sequel. Note that $\operatorname{dim}\left(\Lambda_{n, k}\right)=\left(\begin{array}{l}n \\ k\end{array}\right)$ and that $\operatorname{dim}\left(\Lambda_{n}\right)=2^{n}$.

For any $1 \leq j \leq n$, we let

$$
c_{n, j}: \Lambda_{n} \longrightarrow \Lambda_{n}
$$

be the so-called creation operator defined by letting $c_{n, j}(\Omega)=e_{j}$, and

$$
c_{n, j}\left(\xi_{1} \wedge \cdots \wedge \xi_{k}\right)=e_{j} \wedge \xi_{1} \wedge \cdots \wedge \xi_{k}, \quad \xi_{1}, \ldots, \xi_{k} \in \ell_{n}^{2} .
$$

Next we denote by $P_{n}: \Lambda_{n} \rightarrow \Lambda_{n}$ the orthogonal projection onto the space

$$
\Lambda_{n}^{\text {even }}=\underset{\substack{0 \leq k \leq n \\ k \text { even }}}{\oplus} \Lambda_{n, k}
$$

generated by tensor products of even rank. Following [2, p. 24], we let

$$
x_{n, j}=c_{n, j} P_{n} \quad \text { and } \quad \widetilde{x}_{n, j}=c_{n, j}^{*} P_{n}
$$

be the restrictions of $c_{n, j}$ and $c_{n, j}^{*}$ to $\Lambda_{n}^{\text {even }}$ for any $1 \leq j \leq n$. Then we set

$$
A H_{n}=\operatorname{Span}\left\{x_{n, j}, \widetilde{x}_{n, j}: j=1, \ldots, n\right\} .
$$

This is a $2 n$-dimensional operator space. Next we let

$$
B H_{n}=\left\{x^{*}: x \in A H_{n}\right\}=\operatorname{Span}\left\{x_{n, j}^{*}, \widetilde{x}_{n, j}^{*}: j=1, \ldots, n\right\}
$$

be the adjoint space of $A H_{n}$. Note that $c_{n, j}=P_{n} c_{n, j}+c_{n, j} P_{n}$. Consequently,

$$
\tilde{x}_{n, j}^{*}=c_{n, j}\left(I d-P_{n}\right) \quad \text { and } \quad x_{n, j}^{*}=c_{n, j}^{*}\left(I d-P_{n}\right)
$$

are the restrictions of $c_{n, j}$ and $c_{n, j}^{*}$ to the space

$$
\Lambda_{n}^{\text {odd }}=\Lambda_{n} \ominus \Lambda_{n}^{\text {even }}=\underset{\substack{0 \leq k \leq n \\ k \text { odd }}}{\oplus} \Lambda_{n, k}
$$

generated by the tensor products of odd rank.

In the sequel we let $A H_{n}^{p}$ and $B H_{n}^{p}$ denote the spaces $A H_{n}$ and $B H_{n}$, respectively, regarded as subspaces of $S^{p}\left(\Lambda_{n}\right)$.

Let $\kappa: A H_{n} \rightarrow B H_{n}$ be the exchange map defined by letting

$$
\kappa\left(x_{n, j}\right)=\widetilde{x}_{n, j}^{*} \quad \text { and } \quad \kappa\left(\widetilde{x}_{n, j}\right)=x_{n, j}^{*}, \quad j=1, \ldots, n .
$$

It follows from the calculations in 2, Chap. 2] that $\kappa$ is an isometry from $A H_{n}^{p}$ onto $B H_{n}^{p}$. (An explicit proof of this fact will be given in Section 5; see Remark 5.12.) For any operators $a_{1} \in S^{p}\left(H_{1}\right)$ and $a_{2} \in S^{p}\left(H_{2}\right)$, with $\left(a_{1}, a_{2}\right) \neq(0,0)$, we will consider

$$
Z=\left\{\left(x \otimes a_{1}, \kappa(x) \otimes a_{2}\right): x \in A H_{n}^{p}\right\} \subset S^{p}\left(\Lambda_{n} \stackrel{2}{\otimes} H_{1}\right) \stackrel{p}{\oplus} S^{p}\left(\Lambda_{n} \stackrel{2}{\otimes} H_{2}\right) .
$$


According to [2, Prop. 2.9], this space is 1-complemented in the $p$-direct sum of $S^{p}\left(\Lambda_{n} \stackrel{2}{\otimes} H_{1}\right)$ and $S^{p}\left(\Lambda_{n} \stackrel{2}{\otimes} H_{2}\right)$.

Now following [2, p. 33] we consider the $(2 n-1)$-dimensional operator space

$$
D A H_{n}=\operatorname{Span}\left\{x_{n, n}+\widetilde{x}_{n, n} ; x_{n, j}, \widetilde{x}_{n, j}: j=1, \ldots, n-1\right\},
$$

and we let $D A H_{n}^{p}$ be that space regarded as a subspace of $S^{p}\left(\Lambda_{n}\right)$. Then for any $a \in S^{p}(H)$, the space $D A H_{n}^{p} \otimes a$ is 1 -complemented in $S^{p}\left(\Lambda_{n} \stackrel{2}{\otimes} H\right)$, by [2, Prop. $2.13]$.

Simple proofs of the above-mentioned 1-complementation results will be given later on in Section 5; see Remark 5.12

Definition 2.7. Let $X \subset S^{p}(\mathcal{H}, \mathcal{K})$ be a finite dimensional space of dimension $N \geq 1$. If $N=2 n-1$ is odd, we say that $X$ is a spinorial space if it is equivalent to a space of the form $D A H_{n}^{p} \otimes a$, for some $a \in S^{p}(H)$. If $N=2 n$ is even, we say that $X$ is a spinorial space if it is equivalent to a space of the form (2.12).

See the end of this section for more on this terminology.

We shall now define a class of finite dimensional Hilbert spaces which are 1complemented subspaces of $S^{p}$. Let $1 \leq k \leq n$. It is clear that the creation operators $c_{n, j}$ map $\Lambda_{n, k-1}$ into $\Lambda_{n, k}$. For any $j=1, \ldots, n$, we let

$$
c_{n, j, k}: \Lambda_{n, k-1} \longrightarrow \Lambda_{n, k}
$$

be the restriction of $c_{n, j}$ to $\Lambda_{n, k-1}$. A quick examination of the definition of the $c_{n, j}$ 's shows that the matrix of $c_{n, j, k}$ in the canonical bases of $\Lambda_{n, k-1}$ and $\Lambda_{n, k}$ has its entries in $\{-1,0,1\}$, with at most one nonzero element on each row and on each column. Moreover the \pm 1 entries appear exactly $\left(\begin{array}{l}n-1 \\ k-1\end{array}\right)$ times. Hence $\left\|c_{n, j, k}\right\|_{p}^{p}=\left(\begin{array}{l}n-1 \\ k-1\end{array}\right)$ for any $j$. We let

$$
H_{n, k}=\operatorname{Span}\left\{c_{n, j, k}: j=1, \ldots, n\right\},
$$

and we let $H_{n, k}^{p}$ be that space regarded as a subspace of $S^{p}\left(\Lambda_{n}\right)$. By [3, Chap. 7], $H_{n, k}^{p}$ is an $n$-dimensional Hilbert space. More precisely, the linear mapping

$$
\varphi_{k}: \ell_{n}^{2} \longrightarrow H_{n, k}^{p}, \quad \varphi_{k}\left(e_{j}\right)=c_{n, j, k}, \quad 1 \leq j \leq n,
$$

is a multiple of an isometry; i.e., $\left[\left(\begin{array}{l}n-1 \\ k-1\end{array}\right)\right]^{-\frac{1}{p}} \varphi_{k}$ is an isometry. We refer the reader to [11, 12, 13 for more on these Hilbert spaces and their operator space properties.

For any operators $a_{1} \in S^{p}\left(H_{1}\right), \ldots, a_{n} \in S^{p}\left(H_{n}\right)$, with $\left(a_{1}, \ldots, a_{n}\right) \neq(0, \ldots, 0)$, we will consider the space

$$
E=\left\{\left(\varphi_{1}(s) \otimes a_{1}, \ldots, \varphi_{n}(s) \otimes a_{n}\right): s \in \ell_{n}^{2}\right\} \subset \underset{1 \leq k \leq n}{\oplus} S^{p}\left(\Lambda_{n} \stackrel{2}{\otimes} H_{k}\right) .
$$

Clearly $E$ is a Hilbert space. Indeed if we assume (after normalisation) that

$$
\sum_{k}\left(\begin{array}{c}
n-1 \\
k-1
\end{array}\right)\left\|a_{k}\right\|_{p}^{p}=1
$$

then the linear mapping $\ell_{n}^{2} \rightarrow E$ taking any $s \in \ell_{n}^{2}$ to $\left(\varphi_{1}(s) \otimes a_{1}, \ldots, \varphi_{n}(s) \otimes a_{n}\right)$ is an isometry. According to [2, Prop. 2.5], the space $E$ is 1-complemented. 
Theorem 2.8 (Arazy-Friedman). Let $\mathcal{H}, \mathcal{K}$ be Hilbert spaces, and let $X \subset S^{p}(\mathcal{H}, \mathcal{K})$ be an indecomposable subspace, with $1 \leq p \neq 2<\infty$. The following are equivalent.

(i) $X$ is 1-complemented in $S^{p}(\mathcal{H}, \mathcal{K})$.

(ii) $X$ is either a space of symmetric matrices, or a space of anti-symmetric matrices (in the sense of Definition 2.5), or a space of rectangular matrices (in the sense of Definition [2.6), or a spinorial space (in the sense of Definition 2.7) of dimension $\geq 5$, or a finite dimensional Hilbertian space equivalent to a space of the form (2.14).

By Lemma 2.3 and the results we have recorded in this section, all the spaces in the list (ii) are 1-complemented. The hard implication '(i) $\Rightarrow$ (ii)' is proved in 3 , Chap. 7] in the case $p>1$ and in [2, Chap. 5] in the case $p=1$.

After reducing to the case of indecomposable spaces, the proof of Theorem 1.1 will mainly consist in showing that the spaces in the list (ii) above are not completely 1-complemented, except the ones which are equivalent to some $S_{I, J}^{p} \otimes a$. This will be achieved in the next three sections.

It should be noticed that the classes of 1-complemented subspaces considered above do not exclude each other. For instance, the Hilbert space $S_{n, 1}^{p}$ is equivalent to $H_{n, 1}^{p}$, whereas $S_{1, n}^{p}$ is equivalent to $H_{n, n}^{p}$. On the other hand, $A H_{1}^{p}=\ell_{2}^{p}$ and it follows from [2, Chap. 2] that $A H_{2}^{p}$ is equivalent to $S_{2}^{p}, A H_{3}^{p}$ is equivalent to $\mathcal{A}_{4}^{p}$, $D A H_{2}^{p}$ is equivalent to $\mathcal{S}_{2}^{p}$ and $\mathcal{A}_{3}^{p}$ is equivalent to $H_{3,2}^{p}$.

Remark 2.9. Suppose that $p>1$. If $X \subset S^{p}(\mathcal{H}, \mathcal{K})$ is 1-complemented, then the contractive projection $P: S^{p}(\mathcal{H}, \mathcal{K}) \rightarrow S^{p}(\mathcal{H}, \mathcal{K})$ whose range is equal to $X$ is unique (see [3, Prop. 1.2]).

This uniqueness property is false in the case $p=1$ (see e.g. [2, p. 36]). However a similar result holds true, as follows. Let $X \subset S^{1}(\mathcal{H}, \mathcal{K})$ be a subspace and let $r \in B(\mathcal{H})$ and $\ell \in B(\mathcal{K})$ be the smallest orthogonal projections such that $\ell x r=x$ for any $x \in X$. Let $H \subset \mathcal{H}$ and $K \subset \mathcal{K}$ be the ranges of $r$ and $\ell$, respectively. Thus $X \subset S^{1}(H, K)$, and $H, K$ are the smallest subspaces of $\mathcal{H}, \mathcal{K}$ with that property. We say that $X$ is nondegenerate if $H=\mathcal{H}$ and $K=\mathcal{K}$. It is proved in [2, Th. 2.15] that if $X$ is 1-complemented and nondegenerate, then the contractive projection $P$ on $S^{1}(\mathcal{H}, \mathcal{K})$ with range equal to $X$ is unique.

Note that $X$ regarded as a subspace of $S^{1}(\mathcal{H}, \mathcal{K})$ is equivalent to $X$ regarded as a subspace of $S^{1}(H, K)$. Thus if we wish to determine whether $X$ is [n]-1complemented (for some $n \geq 1$ ), there is no loss of generality in assuming that $X$ is nondegenerate.

We end this section with some terminology and notions, which play a central role in the work of Arazy-Friedman [2, 3, and some basic facts.

Let $\mathcal{H}, \mathcal{K}$ be Hilbert spaces and let $X \subset B(\mathcal{H}, \mathcal{K})$ be a closed subspace. By definition, $X$ is a $J C^{*}$-triple if $x x^{*} x$ belongs to $X$ for any $x \in X$. Next a linear map $u: X \rightarrow Y$ between two $J C^{*}$-triples $X$ and $Y$ is called a triple homomorphism if $u\left(x x^{*} x\right)=u(x) u(x)^{*} u(x)$ for any $x \in X$. If $u$ is one-to-one, we say that $u$ is a triple monomorphism. If further $u$ is a bijection, then $u^{-1}$ also is a triple homomorphism and we say that $u$ is a triple isomorphism in this case. It is well known that a bijection $u: X \rightarrow Y$ between two $J C^{*}$-triples is an isometry if and only if it is a triple isomorphism (see [7]). We say that $X$ and $Y$ are triple equivalent if there is a triple isomorphism from $X$ onto $Y$. 
We now turn to Cartan factors of types 1-4. We mainly follow [7] (see also [1]). By definition, a Cartan factor of type 1 is a $J C^{*}$-triple which is triple equivalent to some $B(\mathcal{H}, \mathcal{K})$, where $\mathcal{H}, \mathcal{K}$ are Hilbert spaces. Next let $\mathcal{H}$ be a Hilbert space with a distinguished Hilbertian basis, and let $w \mapsto{ }^{t} w$ denote the associated transpose map on $B(\mathcal{H})$. Then the space of anti-symmetric operators

$$
\mathcal{A}(\mathcal{H})=\left\{w \in B(\mathcal{H}):{ }^{t} w=-w\right\}
$$

is a $J C^{*}$-triple, and we call a Cartan factor of type 2 any $J C^{*}$-triple which is triple equivalent to some $\mathcal{A}(\mathcal{H})$. Likewise, the space of symmetric operators

$$
\mathcal{S}(\mathcal{H})=\left\{w \in B(\mathcal{H}):{ }^{t} w=w\right\}
$$

is a $J C^{*}$-triple, and we call a Cartan factor of type 3 any $J C^{*}$-triple which is triple equivalent to some $\mathcal{S}(\mathcal{H})$. Lastly, let $X \subset B(\mathcal{H})$ be a closed subspace such that $x^{*} \in X$ for any $x \in X$ and $x^{2}$ is a scalar multiple of the identity operator for any $x \in X$. Then $X$ is a $J C^{*}$-triple, and we call a Cartan factor of type 4 any $J C^{*}$-triple which is triple equivalent to such a space.

Let $n \geq 2$ be an integer. An $n$-tuple $\left(s_{1}, \ldots, s_{n}\right)$ of operators in some $B(\mathcal{H})$ is called a spin system if each $s_{j}$ is a selfadjoint unitary and

$$
s_{j} s_{j^{\prime}}+s_{j^{\prime}} s_{j}=0, \quad 1 \leq j \neq j^{\prime} \leq n .
$$

In this case, the $n$-dimensional space

$$
X=\operatorname{Span}\left\{s_{1}, \ldots, s_{n}\right\} \subset B(\mathcal{H})
$$

is a Cartan factor of type 4 .

Let $w_{1}, \ldots, w_{n}$ be the operators on $\Lambda_{n}$ defined by

$$
\omega_{j}=c_{n, j}+c_{n, j}^{*}, \quad j=1, \ldots, n .
$$

These operators are called Fermions and they form a spin system (see e.g. [4]). Hence their linear span is an $n$-dimensional Cartan factor of type 4 . It is well known that all $n$-dimensional Cartan factors of type 4 are mutually triple equivalent. Thus the space $\operatorname{Span}\left\{\omega_{1}, \ldots, \omega_{n}\right\}$ is actually a model for such spaces.

It turns out that the spaces $A H_{n}$ and $D A H_{n}$ considered in this section are Cartan factors of type 4 . This will be implicitly shown along with the proof of Theorem 5.11. We refer the reader to 3 for details on this, and for a deeper analysis of the relationship between 1-complemented subspaces of $S^{p}$-spaces and Cartan factors.

In the framework of operator space theory, it is natural to wonder whether a triple isomorphism between Cartan factors is necessarily a complete isometry, that is, if the identification of Cartan factors in the category of $J C^{*}$-triples coincides with their identification as operator spaces. This is not always the case. Indeed, if $\mathcal{H}$ or $\mathcal{K}$ has dimension $\geq 2$, any transposition map $B(\mathcal{H}, \mathcal{K}) \rightarrow B(\mathcal{K}, \mathcal{H})$ is a triple isomorphism which is not completely isometric. This question is more delicate for Cartan factors of types 2-4 and will be discussed further in Section 7 (see in particular Proposition 7.3 and Remark 7.4 .

\section{Elementary computations}

In this section we will treat rectangular matrices, symmetric matrices, and antisymmetric matrices. We will only need elementary matrix computations.

Proposition 3.1. Let $X \subset S^{p}(\mathcal{H}, \mathcal{K})$ be a space of rectangular matrices, and assume that $1 \leq p \neq 2<\infty$. 
(1) If $X$ is [2]-1-complemented in $S^{p}(\mathcal{H}, \mathcal{K})$, then there exist two index sets $I, J$, a Hilbert space $H$ and an operator $a \in S^{p}(H)$ such that $X$ is equivalent to $S_{I, J}^{p} \otimes a$.

(2) If $X$ is equivalent to a space of the form $S_{I, J}^{p} \otimes a$, then $X$ is completely 1-complemented in $S^{p}(\mathcal{H}, \mathcal{K})$.

Proof. Part (2) is obvious by Lemma 2.3 To prove (1), it suffices by Lemma 2.3 again to consider index sets $I, J$ with $(I, J) \neq(1,1)$ and to show that for any operators $a_{1} \in S^{p}\left(H_{1}\right), a_{2} \in S^{p}\left(H_{2}\right)$, the space defined by (2.8) is [2]-1complemented only if $a_{1}=0$ or $a_{2}=0$. We will use matrix notation as introduced in Section 2. We assume that $\left\|a_{1}\right\|_{p}^{p}+\left\|a_{2}\right\|_{p}^{p}=1$ and we let $t=\left\|a_{1}\right\|_{p}^{p}$.

Let us write $Y_{I, J}$ for the space defined by (2.8). For any $I^{\prime} \subset I$ and $J^{\prime} \subset J$, we have a natural inclusion $Y_{I^{\prime}, J^{\prime}} \subset Y_{I, J}$. Then $Y_{I^{\prime}, J^{\prime}}$ is clearly completely 1complemented in $Y_{I, J}$. Hence without loss of generality we can assume that $I=2$ and $J=1$.

Assume that $Y=Y_{2,1}$ is [2]-1-complemented. Then by Remark 2.9, the projection $P$ on $Y_{1} \stackrel{p}{\oplus} Y_{2}$ defined by (2.9) is [2]-contractive. According to (2.6), we have an isometric identification

$$
S_{2}^{p} \stackrel{p}{\otimes}\left(Y_{1} \stackrel{p}{\oplus} Y_{2}\right)=\left[\left(S_{2}^{p} \stackrel{p}{\otimes} S_{2,1}^{p}\right) \otimes a_{1}\right] \stackrel{p}{\oplus}\left[\left(S_{2}^{p} \stackrel{p}{\otimes} S_{1,2}^{p}\right) \otimes a_{2}\right],
$$

and for any $z_{1} \in S_{2}^{p} \stackrel{p}{\otimes} S_{2,1}^{p} \simeq S_{4,2}^{p}$ and $z_{2} \in S_{2}^{p} \stackrel{p}{\otimes} S_{1,2}^{p} \simeq S_{2,4}^{p}$, we have

$$
\left(I_{S_{2}^{p}} \otimes P\right)\left(\left[\begin{array}{cc}
z_{1} & 0 \\
0 & z_{2}
\end{array}\right]\right)=\left[\begin{array}{cc}
t z_{1}+(1-t)\left[I_{S_{2}^{p}} \otimes \sigma^{-1}\right]\left(z_{2}\right) & 0 \\
0 & t\left[I_{S_{2}^{p}} \otimes \sigma\right]\left(z_{1}\right)+(1-t) z_{2}
\end{array}\right] .
$$

Assume first that $2<p<\infty$. For any positive angle $\theta>0$, consider

$$
z_{1}(\theta)=\left[\begin{array}{cc}
(\cos (\theta))^{\frac{2}{p}} & 0 \\
0 & 0 \\
0 & (\sin (\theta))^{\frac{2}{p}} \\
0 & 0
\end{array}\right] \text { and } z_{2}(\theta)=\left[\begin{array}{cccc}
(\cos (\theta))^{\frac{2}{p}} & 0 & 0 & 0 \\
0 & 0 & (\sin (\theta))^{\frac{2}{p}} & 0
\end{array}\right] \text {. }
$$

Then we have

$$
\left\|z_{1}(\theta)\right\|_{p}^{p}=\left\|z_{2}(\theta)\right\|_{p}^{p}=\cos (\theta)^{2}+\sin (\theta)^{2}=1
$$

hence

$$
\left\|\left[\begin{array}{cc}
z_{1}(\theta) & 0 \\
0 & z_{2}(\theta)
\end{array}\right]\right\|_{S_{2}^{p} \stackrel{p}{\otimes}\left(Y_{1}^{\oplus} \stackrel{p}{\oplus} Y_{2}\right)}=1 .
$$

Applying the transpose map $\sigma: S_{2,1}^{p} \rightarrow S_{1,2}^{p}$ and its inverse, we find that

$$
\left[I_{S_{2}^{p}} \otimes \sigma\right]\left(z_{1}(\theta)\right)=\left[\begin{array}{cccc}
(\cos (\theta))^{\frac{2}{p}} & 0 & 0 & (\sin (\theta))^{\frac{2}{p}} \\
0 & 0 & 0 & 0
\end{array}\right]
$$

and that

$$
\left[I_{S_{2}^{p}} \otimes \sigma^{-1}\right]\left(z_{2}(\theta)\right)=\left[\begin{array}{cc}
(\cos (\theta))^{\frac{2}{p}} & 0 \\
0 & 0 \\
0 & 0 \\
(\sin (\theta))^{\frac{2}{p}} & 0
\end{array}\right]
$$


Consequently, we have

$$
t z_{1}(\theta)+(1-t)\left[I_{S_{2}^{p}} \otimes \sigma^{-1}\right]\left(z_{2}(\theta)\right)=\left[\begin{array}{cc}
(\cos (\theta))^{\frac{2}{p}} & 0 \\
0 & 0 \\
0 & t(\sin (\theta))^{\frac{2}{p}} \\
(1-t)(\sin (\theta))^{\frac{2}{p}} & 0
\end{array}\right],
$$

whereas

$t\left[I_{S_{2}^{p}} \otimes \sigma\right]\left(z_{1}(\theta)\right)+(1-t) z_{2}(\theta)=\left[\begin{array}{cccc}(\cos (\theta))^{\frac{2}{p}} & 0 & 0 & t(\sin (\theta))^{\frac{2}{p}} \\ 0 & 0 & (1-t)(\sin (\theta))^{\frac{2}{p}} & 0\end{array}\right]$.

Thus

$$
\begin{aligned}
\left\|t z_{1}(\theta)+(1-t)\left[I_{S_{2}^{p}} \otimes \sigma^{-1}\right]\left(z_{2}(\theta)\right)\right\|_{p} & \geq\left\|\left[\begin{array}{c}
(\cos (\theta))^{\frac{2}{p}} \\
(1-t)(\sin (\theta))^{\frac{2}{p}}
\end{array}\right]\right\|_{S_{2,1}^{p}} \\
& =\left((\cos (\theta))^{\frac{4}{p}}+(1-t)^{2}(\sin (\theta))^{\frac{4}{p}}\right)^{\frac{1}{2}} .
\end{aligned}
$$

Likewise,

$$
\left\|t\left[I_{S_{2}^{p}} \otimes \sigma\right]\left(z_{1}(\theta)\right)+(1-t) z_{2}(\theta)\right\|_{p} \geq\left((\cos (\theta))^{\frac{4}{p}}+t^{2}(\sin (\theta))^{\frac{4}{p}}\right)^{\frac{1}{2}} .
$$

Using (3.2), these estimates imply that

$$
\|P\|_{2}^{p} \geq t\left((\cos (\theta))^{\frac{4}{p}}+(1-t)^{2}(\sin (\theta))^{\frac{4}{p}}\right)^{\frac{p}{2}}+(1-t)\left((\cos (\theta))^{\frac{4}{p}}+t^{2}(\sin (\theta))^{\frac{4}{p}}\right)^{\frac{p}{2}} .
$$

Since $p>2$, we have $\frac{4}{p}<2$. Consequently,

$$
(\cos (\theta))^{\frac{4}{p}}=1+o\left(\theta^{\frac{4}{p}}\right) \quad \text { and } \quad(\sin (\theta))^{\frac{4}{p}}=\theta^{\frac{4}{p}}+o\left(\theta^{\frac{4}{p}}\right)
$$

on a (positive) neighborhood of zero. Hence

$$
\begin{aligned}
1=\|P\|_{2}^{p} & \geq t\left(1+(1-t)^{2} \theta^{\frac{4}{p}}+o\left(\theta^{\frac{4}{p}}\right)\right)^{\frac{p}{2}}+(1-t)\left(1+t^{2} \theta^{\frac{4}{p}}+o\left(\theta^{\frac{4}{p}}\right)\right)^{\frac{p}{2}} \\
& \geq 1+\frac{p}{2}\left(t(1-t)^{2}+(1-t) t^{2}\right) \theta^{\frac{4}{p}}+o\left(\theta^{\frac{4}{p}}\right) \\
& =1+\frac{p}{2} t(1-t) \theta^{\frac{4}{p}}+o\left(\theta^{\frac{4}{p}}\right) .
\end{aligned}
$$

This implies that $t(1-t)=0$; that is, $a_{1}=0$ or $a_{2}=0$.

The case when $1<p<2$ can be treated by duality, or by a direct similar argument. Indeed, let $p^{\prime}=(p-1) / p>2$ be the conjugate number of $p$ and apply (3.1) with

$$
z_{1}=\left[\begin{array}{cc}
(\cos (\theta))^{\frac{2}{p^{\prime}}} & 0 \\
0 & 0 \\
0 & 0 \\
(\sin (\theta))^{\frac{2}{p^{\prime}}} & 0
\end{array}\right] \quad \text { and } \quad z_{2}=\left[\begin{array}{cccc}
(\cos (\theta))^{\frac{2}{p^{\prime}}} & 0 & 0 & (\sin (\theta))^{\frac{2}{p^{\prime}}} \\
0 & 0 & 0 & 0
\end{array}\right] .
$$

Then we find that

$$
\left\|\left[\begin{array}{cc}
z_{1} & 0 \\
0 & z_{2}
\end{array}\right]\right\|=1+\frac{1}{2} \theta^{\frac{4}{p^{\prime}}}+o\left(\theta^{\frac{4}{p^{\prime}}}\right)
$$

and that

$$
\left\|\left(I_{S_{2}^{p}} \otimes P\right)\left[\begin{array}{cc}
z_{1} & 0 \\
0 & z_{2}
\end{array}\right]\right\| \geq 1+\frac{1}{p}\left(t(1-t)^{p}+t^{p}(1-t)\right) \theta^{\frac{2 p}{p^{\prime}}}+o\left(\theta^{\frac{2 p}{p^{\prime}}}\right) .
$$

Since $\frac{2 p}{p^{\prime}}<\frac{4}{p^{\prime}}$, we deduce that $t=0$ or $t=1$ if $P$ is [2]-contractive. 
The case $p=1$ has a similar proof, with

$$
z_{1}=\left[\begin{array}{cc}
1 & 0 \\
0 & 0 \\
0 & 0 \\
\theta & 0
\end{array}\right] \quad \text { and } \quad z_{2}=\left[\begin{array}{cccc}
1 & 0 & 0 & \theta \\
0 & 0 & 0 & 0
\end{array}\right]
$$

Proposition 3.2. Let $X \subset S^{p}(\mathcal{H}, \mathcal{K})$ and assume that $1 \leq p \neq 2<\infty$ and $\operatorname{dim}(X)>1$. If $X$ is either a space of symmetric matrices or a space of antisymmetric matrices, then $X$ is not [2]-1-complemented.

Proof. By Lemma 2.3, it suffices to show that for any $a \in S^{p}(H) \backslash\{0\}$, the space $\mathcal{S}_{I}^{p} \otimes a$ is not [2]-1-complemented, unless $I=1$, and that the space $\mathcal{A}_{I}^{p} \otimes a$ is not [2]-1-complemented, unless $I=1$ or 2 . Using Remark 2.9 and an obvious reduction, this amounts to showing that the contractive projections

$$
P_{s}: S_{2}^{p} \longrightarrow S_{2}^{p} \quad \text { and } \quad P_{a}: S_{3}^{p} \longrightarrow S_{3}^{p}
$$

given by (2.7) are not [2]-contractive.

The subspace

$$
\left\{\left[\begin{array}{ccc}
0 & s_{12} & s_{13} \\
s_{21} & 0 & 0 \\
s_{31} & 0 & 0
\end{array}\right]: s_{12}, s_{13}, s_{21}, s_{31} \in \mathbb{C}\right\} \subset S_{3}^{p}
$$

is completely isometric to $S_{2,1}^{p} \stackrel{p}{\oplus} S_{1,2}^{p}$, and

$$
P_{a}\left(\left[\begin{array}{ccc}
0 & s_{12} & s_{13} \\
s_{21} & 0 & 0 \\
s_{31} & 0 & 0
\end{array}\right]\right)=\frac{1}{2}\left[\begin{array}{ccc}
0 & s_{12}-s_{21} & s_{13}-s_{31} \\
s_{21}-s_{12} & 0 & 0 \\
s_{31}-s_{13} & 0 & 0
\end{array}\right] .
$$

Hence

$$
\left\|I_{S_{2}^{p}} \otimes P_{a}\right\| \geq\left\|I_{S_{2}^{p}} \otimes P\right\|,
$$

where $P$ is the projection defined by (2.9) in the case when $I=2, J=1$, and $\left\|a_{1}\right\|=\left\|a_{2}\right\|$. It follows from the proof of Proposition 3.1 that this projection is not [2]-contractive. Thus $P_{a}$ is not [2]-contractive either.

The fact that $P_{s}$ is not [2]-contractive on $S_{2}^{p}$ also follows from the proof of Proposition 3.1. We skip the details.

\section{Finite dimensional Hilbertian subspaces}

In this section we will treat finite dimensional Hilbertian 1-complemented subspaces of $S^{p}$-spaces. We use the notation introduced in Section 2. Let $n \geq 2$ be an integer. For any contraction $T: \ell_{n}^{2} \rightarrow \ell_{n}^{2}$, we let $F(T): \Lambda_{n} \rightarrow \Lambda_{n}$ be the linear mapping defined by $F(T) \Omega=\Omega$ and

$$
F(T)\left(\xi_{1} \wedge \cdots \wedge \xi_{k}\right)=T\left(\xi_{1}\right) \wedge \cdots \wedge T\left(\xi_{k}\right), \quad \xi_{1}, \ldots, \xi_{k} \in \ell_{n}^{2} .
$$

It is well known that $F(T)$ is a contraction (the construction $T \mapsto F(T)$ is called the second quantization). Note that $F\left(T_{1} T_{2}\right)=F\left(T_{1}\right) F\left(T_{2}\right)$ for any two contractions $T_{1}, T_{2}$ of $\ell_{n}^{2}$. Thus $F(U)$ is a unitary if $U$ is a unitary, and we have $F(U)^{*}=F\left(U^{*}\right)$ in this case. We will need the following observation of independent interest. 
Lemma 4.1. Let $U: \ell_{n}^{2} \rightarrow \ell_{n}^{2}$ be a unitary operator, and let $\widehat{U}: B\left(\Lambda_{n}\right) \rightarrow B\left(\Lambda_{n}\right)$ be defined by

$$
\widehat{U}(W)=F(U) W F(U)^{*}, \quad W \in B\left(\Lambda_{n}\right) .
$$

Then for any $1 \leq k \leq n, \widehat{U}\left(H_{n, k}\right) \subset H_{n, k}$ and the restriction of $\widehat{U}$ to $H_{n, k}$ coincides with $U$. (More precisely, $U=\varphi_{k}^{-1} \widehat{U} \varphi_{k}$, where $\varphi_{k}: \ell_{n}^{2} \rightarrow H_{n, k}$ is defined by (2.13).)

Proof. It is clear that $\widehat{U}$ maps $B\left(\Lambda_{n, k-1}, \Lambda_{n, k}\right)$ into itself. Assume for simplicity that $k \geq 2$ (the case $k=1$ is similar). Let $1 \leq j \leq n$, and let $\xi_{1}, \ldots, \xi_{k-1} \in \ell_{n}^{2}$. Then

$$
\begin{aligned}
{\left[\widehat{U}\left(c_{n, j, k}\right)\right]\left(\xi_{1} \wedge \cdots \wedge \xi_{k-1}\right) } & =F(U) c_{n, j, k} F(U)^{*}\left(\xi_{1} \wedge \cdots \wedge \xi_{k-1}\right) \\
& =F(U) c_{n, j, k}\left(U^{*}\left(\xi_{1}\right) \wedge \cdots \wedge U^{*}\left(\xi_{k-1}\right)\right) \\
& =F(U)\left(e_{j} \wedge U^{*}\left(\xi_{1}\right) \wedge \cdots \wedge U^{*}\left(\xi_{k-1}\right)\right) \\
& =U\left(e_{j}\right) \wedge \xi_{1} \wedge \cdots \wedge \xi_{k-1},
\end{aligned}
$$

since $U U^{*}=I_{\ell_{n}^{2}}$. This yields the result.

It was proved in [12, Thm. 1] that for any $1 \leq k \leq n, H_{n, k} \subset B\left(\Lambda_{n}\right)$ is a homogeneous operator space. Using [18, Prop. 9.2.1], this result readily follows from the above lemma. The latter implies that $H_{n, k}^{p} \subset S^{p}\left(\Lambda_{n}\right)$ is homogeneous as well.

Proposition 4.2. Assume that $1 \leq p \neq 2<\infty$ and let $a_{1} \in S^{p}\left(H_{1}\right), \ldots, a_{n} \in$ $S^{p}\left(H_{n}\right)$, with $\left(a_{1}, \ldots, a_{n}\right) \neq(0, \ldots, 0)$. The $n$-dimensional Hilbert space $E$ defined by (2.14) is [2]-1-complemented if and only if

$$
a_{2}=\cdots=a_{n}=0 \quad \text { or } \quad a_{1}=\cdots=a_{n-1}=0 .
$$

Proof. The 'if' part is clear. If for example $a_{2}=\cdots=a_{n}=0$, then

$$
E=H_{n, 1}^{p} \otimes a_{1} \sim S_{n, 1}^{p} \otimes a_{1}
$$

hence $E$ is completely complemented.

We shall now prove the 'only if' part. We assume that $E$ is [2]-1-complemented. We will somehow reduce to the case when $\operatorname{dim}(E)=2$. Let us apply the second quantization to the unitary

$$
U=\left[\begin{array}{ccccc}
1 & & & & \\
& 1 & & (0) & \\
& & -1 & & \\
& (0) & & \ddots & \\
& & & & -1
\end{array}\right]: \ell_{n}^{2} \longrightarrow \ell_{n}^{2} .
$$

Since $U^{2}$ is the identity on $\ell_{n}^{2}$, we have $F(U)^{2}=I_{\Lambda_{n}}$; hence $\widehat{U}^{2}$ is the identity operator on $B\left(\Lambda_{n}\right)$. We set

$$
\Delta=\frac{I d+\widehat{U}}{2} .
$$

Then $\Delta$ is a projection. Moreover $\widehat{U}$ is a complete contraction on $S^{p}\left(\Lambda_{n}\right)$ by Lemma 2.2. hence $\Delta: S^{p}\left(\Lambda_{n}\right) \rightarrow S^{p}\left(\Lambda_{n}\right)$ is a complete contraction as well. We let

$$
\Delta^{\oplus n}: \underset{1 \leq k \leq n}{\oplus} S^{p}\left(\Lambda_{n}\right) \otimes a_{k} \longrightarrow \underset{1 \leq k \leq n}{\stackrel{p}{\oplus}} S^{p}\left(\Lambda_{n}\right) \otimes a_{k}
$$


be the amplification of $\Delta$ taking $\left(z_{1} \otimes a_{1}, \ldots, z_{n} \otimes a_{n}\right)$ to $\left(\Delta\left(z_{1}\right) \otimes a_{1}, \ldots, \Delta\left(z_{n}\right) \otimes a_{n}\right)$ for any $z_{1}, \ldots, z_{n}$ in $S^{p}\left(\Lambda_{n}\right)$. Clearly, $\Delta^{\oplus n}$ also is a completely contractive projection.

It follows from Lemma 4.1 that for any $1 \leq k \leq n, \Delta$ maps $H_{n, k}$ into itself, and that

$$
\begin{aligned}
\Delta\left(\varphi_{k}(s)\right) & =\varphi_{k}\left(\left\langle s, e_{1}\right\rangle e_{1}+\left\langle s, e_{2}\right\rangle e_{2}\right) \\
& =\left\langle s, e_{1}\right\rangle c_{n, 1, k}+\left\langle s, e_{2}\right\rangle c_{n, 2, k}, \quad s \in \ell_{n}^{2} .
\end{aligned}
$$

Thus $\Delta^{\oplus n}$ maps $E$ into itself, and

$\Delta^{\oplus n}(E)=\left\{\left(\left(s_{1} c_{n, 1,1}+s_{2} c_{n, 2,1}\right) \otimes a_{1}, \ldots,\left(s_{1} c_{n, 1, n}+s_{2} c_{n, 2, n}\right) \otimes a_{n}\right): s_{1}, s_{2} \in \mathbb{C}\right\}$

is completely 1-complemented in $E$. Therefore, the 2-dimensional Hilbert space $\Delta^{\oplus n}(E)$ is [2]-1-complemented in the $p$-direct sum of the $S^{p}\left(\Lambda_{n}\right) \otimes a_{k}$ 's.

Let $1 \leq k \leq n$. Given any $s_{1}, s_{2} \in \mathbb{C}$, let us look at the matrix $M_{k}\left(s_{1}, s_{2}\right)$ of the operator $s_{1} c_{n, 1, k}+s_{2} c_{n, 2, k}$ in the canonical bases $\left\{e_{B}:|B|=k-1\right\}$ and $\left\{e_{A}:|A|=k\right\}$ of $\Lambda_{n, k-1}$ and $\Lambda_{n, k}$ (see Section 2). We call $m_{A, B}$ the entries of this matrix. If $1 \notin B$ and $2 \notin B$, then $m_{A_{1}, B}=s_{1}$ and $m_{A_{2}, B}=s_{2}$, where $A_{1}=B \cup\{1\}$ and $A_{2}=B \cup\{2\}$. All other entries in the column indexed by $B$ are equal to 0 . This case occurs $\left(\begin{array}{c}n-2 \\ k-1\end{array}\right)$ times. Otherwise, that is, if $1 \in B$ or $2 \in B$, then the column indexed by $B$ has at most one nonzero entry. Likewise if $\{1,2\} \subset A$, then $m_{A, B_{1}}=s_{1}$ and $m_{A, B_{2}}=-s_{2}$, where $B_{1}=A \backslash\{1\}$ and $B_{2}=A \backslash\{2\}$. All other entries in the row indexed by $A$ are equal to 0 . This case occurs $\left(\begin{array}{l}n-2 \\ k-2\end{array}\right)$ times. Otherwise, that is, if $1 \notin A$ or $2 \notin A$, then the row indexed by $A$ has at most one nonzero entry. Furthermore the submatrices

$$
\left[\begin{array}{l}
s_{1} \\
s_{2}
\end{array}\right] \quad \text { and } \quad\left[\begin{array}{ll}
s_{1} & -s_{2}
\end{array}\right]
$$

appearing in $M_{k}\left(s_{1}, s_{2}\right)$ are 'orthogonal' to each other. Namely if $m_{A_{1}, B}=s_{1}$, $m_{A_{2}, B}=s_{2}, m_{A, B_{1}}=s_{1}$ and $m_{A, B_{2}}=-s_{2}$, then $A$ is both different from $A_{1}$ and $A_{2}$ and $B$ is both different from $B_{1}$ and $B_{2}$. Consequently for an appropriate ordering of the canonical bases of $\Lambda_{n, k-1}$ and $\Lambda_{n, k}$, we have a block diagonal representation

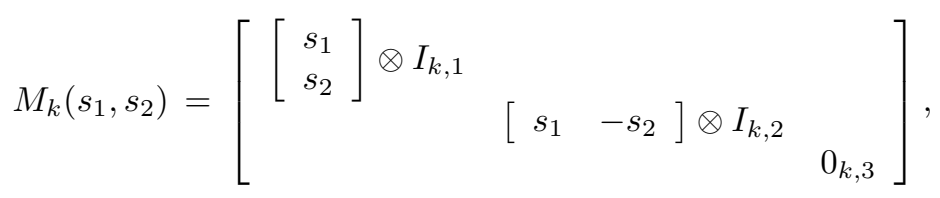

where $I_{k, 1}$ is the unit of the square matrices of size $\left(\begin{array}{c}n-2 \\ k-1\end{array}\right), I_{k, 2}$ is the unit of the square matrices of size $\left(\begin{array}{l}n-2 \\ k-2\end{array}\right)$, and $0_{k, 3}$ is the zero rectangular matrix of size $\left(\begin{array}{c}n-2 \\ k\end{array}\right) \times$ $\left(\begin{array}{l}n-2 \\ k-3\end{array}\right)$. We deduce that there exist two operators $b_{1} \in S^{p}\left(K_{1}\right)$ and $b_{2} \in S^{p}\left(K_{2}\right)$ (defined on large enough Hilbert spaces $K_{1}, K_{2}$ ) such that

$$
\Delta^{\oplus n}(E) \sim Y:=\left\{\left(\left[\begin{array}{l}
s_{1} \\
s_{2}
\end{array}\right] \otimes b_{1},\left[\begin{array}{ll}
s_{1} & -s_{2}
\end{array}\right] \otimes b_{2}\right): s_{1}, s_{2} \in \mathbb{C}\right\}
$$

and

$$
\left\|b_{1}\right\|^{p}=\sum_{k=1}^{n}\left(\begin{array}{c}
n-2 \\
k-1
\end{array}\right)\left\|a_{k}\right\|_{p}^{p} \quad \text { and } \quad\left\|b_{2}\right\|^{p}=\sum_{k=1}^{n}\left(\begin{array}{c}
n-2 \\
k-2
\end{array}\right)\left\|a_{k}\right\|_{p}^{p} .
$$


By Lemma 2.3. $Y$ is [2]-1-complemented in $S^{p}\left(\ell_{2}^{2}\left(K_{1}\right), K_{1}\right) \stackrel{p}{\oplus} S^{p}\left(K_{2}, \ell_{2}^{2}\left(K_{2}\right)\right)$, which implies by Proposition 3.1 that $b_{1}=0$ or $b_{2}=0$. For any $1 \leq k \leq n-1$, we have $\left(\begin{array}{l}n-2 \\ k-1\end{array}\right) \neq 0$. Hence in the case when $b_{1}=0$, we have $a_{1}=\cdots=a_{n-1}=0$ by (4.1). Likewise in the case when $b_{2}=0$, we have $a_{2}=\cdots=a_{n}=0$.

\section{SpinORIAL Subspaces}

In this section we will prove that spinorial spaces (as defined in Definition 2.7) of dimension $\geq 5$ cannot be [2]-1-complemented. As an intermediate step of independent interest, we will consider a variant of these spaces, using the Fermions and Clifford algebras. The necessary background on these topics can be found in 4, 20].

We need a few simple facts about noncommutative $L^{p}$-spaces and their completely bounded maps. The following definitions extend those given in Section 2 for the Schatten spaces. If $M$ is any semifinite von Neumann algebra equipped with a normal semifinite faithful trace $\varphi$, and $1 \leq p<\infty$, we let $L^{p}(M, \varphi)$ (or simply $\left.L^{p}(M)\right)$ denote the associated noncommutative $L^{p}$-space. Recall that if we let

$$
\|x\|_{p}=\left(\varphi\left(|x|^{p}\right)\right)^{\frac{1}{p}}, \quad x \in M
$$

then $L^{p}(M, \varphi)$ is the completion of the space $\left\{x \in M:\|x\|_{p}<\infty\right\}$ equipped with \|\|$_{p}$. See e.g. [19] for information on these spaces. If $\left(M_{1}, \varphi_{1}\right)$ and $\left(M_{2}, \varphi_{2}\right)$ are two semifinite von Neumann algebras, let $M_{1} \bar{\otimes} M_{2}$ denote the von Neumann algebra tensor product and let $\varphi_{1} \bar{\otimes} \varphi_{2}$ denote the associated semifinite faithful trace. Then

$$
L^{p}\left(M_{1}, \varphi_{1}\right) \otimes L^{p}\left(M_{2}, \varphi_{2}\right) \subset L^{p}\left(M_{1} \bar{\otimes} M_{2}, \varphi_{1} \bar{\otimes} \varphi_{2}\right)
$$

is a dense subspace. For any closed subspace $X \subset L^{p}\left(M_{2}, \varphi_{2}\right)$, we denote by

$$
L^{p}\left(M_{1}, \varphi_{1}\right) \stackrel{p}{\otimes} X \subset L^{p}\left(M_{1} \bar{\otimes} M_{2}, \varphi_{1} \bar{\otimes} \varphi_{2}\right)
$$

the closure of $L^{p}\left(M_{1}, \varphi_{1}\right) \otimes X$ in $L^{p}\left(M_{1} \bar{\otimes} M_{2}, \varphi_{1} \bar{\otimes} \varphi_{2}\right)$.

If $u: X \rightarrow Y$ is any bounded linear map between two subspaces of noncommutative $L^{p}$-spaces, we define $\|u\|_{n}$ and $\|u\|_{c b}$ by (2.1) and (2.3). Then the terminology introduced in the second paragraph of Section 2 also extends to this context. Again we refer to [17] for the connections with operator space theory and further information. We will use repeatedly the following well known easy fact.

Lemma 5.1. Assume that $\left(M_{1}, \varphi_{1}\right)$ and $\left(M_{2}, \varphi_{2}\right)$ are finite von Neumann algebras and let $\pi: M_{1} \rightarrow M_{2}$ be a one-to-one $*$-representation. Let $\delta>0$ be a constant and assume that $\varphi_{2}(\pi(x))=\delta \varphi_{1}(x)$ for any $x \in M_{1}$. Then for any $1 \leq p<\infty, \delta^{-p} \pi$ (uniquely) extends to a complete isometry from $L^{p}\left(M_{1}\right)$ into $L^{p}\left(M_{2}\right)$.

For any Hilbert space $\mathcal{H}$ we write $\operatorname{tr}$ for the usual trace on $B(\mathcal{H})$. In the sequel, the semifinite von Neumann algebras we will meet will be either finite dimensional ones or the Schatten spaces $S^{p}(\mathcal{H})=L^{p}(B(\mathcal{H}), t r)$.

Let $N \geq 1$ be an integer. For convenience we write 1 for the identity operator on $B\left(\Lambda_{N}\right)$. Recall that the Fermions $\omega_{j}=c_{N, j}+c_{N, j}^{*} \in B\left(\Lambda_{n}\right)$ are selfadjoint unitaries which anti-commute, that is,

$$
\forall j, \quad \omega_{j}^{2}=1 \text { and } \omega_{j}^{*}=\omega_{j} ; \quad \forall j \neq j^{\prime}, \quad \omega_{j} \omega_{j^{\prime}}=-\omega_{j^{\prime}} \omega_{j} .
$$


These properties will be used throughout without any further comments. As an immediate consequence, we have

$$
\left(\omega_{1} \cdots \omega_{N-1} \omega_{N}\right)^{2}=(-1)^{\frac{N(N-1)}{2}} .
$$

The Clifford algebra with $N$ generators is the $C^{*}$-algebra

$$
\mathcal{C}_{N}=C^{*}\left\langle\omega_{1}, \ldots, \omega_{N}\right\rangle \subset B\left(\Lambda_{N}\right)
$$

generated by the first $N$ Fermions. The dimension of $\mathcal{C}_{N}$ is equal to $2^{N}$. More precisely, for any $A \in \mathcal{P}_{N}$ (the set of all subsets of $\{1, \ldots, N\}$ ), set $\omega_{A}=\omega_{i_{1}} \cdots \omega_{i_{\ell}}$, when $A=\left\{i_{1}, \ldots, i_{\ell}\right\}$ and $i_{1}<\cdots<i_{\ell}$. By convention, $\omega_{\emptyset}=1$. Then $\left\{\omega_{A}: A \in\right.$ $\left.\mathcal{P}_{N}\right\}$ is a basis of $\mathcal{C}_{N}$.

Recall that $\Omega \in \Lambda_{N}$ denotes the vacuum vector. The functional $\operatorname{Tr}: \mathcal{C}_{N} \rightarrow \mathbb{C}$ defined by

$$
\operatorname{Tr}(x)=\langle x(\Omega), \Omega\rangle, \quad x \in \mathcal{C}_{N},
$$

is a normalized trace on $\mathcal{C}_{N}$. For $1 \leq p<\infty$, we let $L^{p}\left(\mathcal{C}_{N}\right)$ denote the associated noncommutative $L^{p}$-space. In the sequel, by an orthogonal projection $\mathcal{C}_{N} \rightarrow \mathcal{C}_{N}$, we will simply mean a projection which is orthogonal on the Hilbert space $L^{2}\left(\mathcal{C}_{N}\right)$. It turns out (easy to check) that $\left\{\omega_{A}: A \in \mathcal{P}_{N}\right\}$ is an orthonormal basis of $L^{2}\left(\mathcal{C}_{N}\right)$.

We will focus on the operator space

$$
E_{N}=\operatorname{Span}\left\{1, \omega_{1}, \ldots, \omega_{N}\right\} \subset \mathcal{C}_{N} .
$$

Lemma 5.2. Let $P: \mathcal{C}_{N} \rightarrow \mathcal{C}_{N}$ be the orthogonal projection onto $E_{N}$. Then

$$
\left\|P: L^{p}\left(\mathcal{C}_{N}\right) \longrightarrow L^{p}\left(\mathcal{C}_{N}\right)\right\|=1
$$

for any $1 \leq p \leq \infty$.

Proof. We will first show that $P$ is positive. Let $x \in \mathcal{C}_{N}$ with $x \geq 0$. In particular $x$ is selfadjoint. Since $E_{N}$ and $E_{N}^{\perp}$ are both selfadjoint subspaces of $\mathcal{C}_{N}$, this implies that $P(x)$ is selfadjoint as well. Thus there exist real numbers $\alpha, \beta_{1}, \ldots, \beta_{N}$ such that

$$
P(x)=\alpha 1+\sum_{j=1}^{N} \beta_{j} \omega_{j} .
$$

Let

$$
\beta=\left(\sum_{j=1}^{N} \beta_{j}^{2}\right)^{\frac{1}{2}} \quad \text { and } \quad \omega=\beta^{-1} \sum_{j=1}^{N} \beta_{j} \omega_{j}
$$

if $\beta \neq 0$. Since the $\beta_{j}$ 's are real, we have $\omega^{*}=\omega$ and the anticommutation relations yield

$$
\omega^{2}=\beta^{-2} \sum_{j, j^{\prime}} \beta_{j} \beta_{j^{\prime}} \omega_{j} \omega_{j^{\prime}}=\beta^{-2} \sum_{j} \beta_{j}^{2}=1 .
$$

Thus $\omega$ is a selfadjoint unitary. Let $q_{+}=2^{-1}(1+\omega)$ and $q_{-}=2^{-1}(1-\omega)$. Then $q_{+}$and $q_{-}$are orthogonal projections with sum $q_{+}+q_{-}=1$ and

$$
P(x)=(\alpha+\beta) q_{+}+(\alpha-\beta) q_{-} .
$$

Since $\operatorname{Tr}\left(\omega_{j}\right)=0$ for any $j$, we have $\operatorname{Tr}(\omega)=0$. Hence $\operatorname{Tr}\left(q_{+}\right)=1 / 2$. Consequently,

$$
\operatorname{Tr}\left(x q_{+}\right)=\operatorname{Tr}\left(x P\left(q_{+}\right)\right)=\operatorname{Tr}\left(P(x) q_{+}\right)=\frac{\alpha+\beta}{2} .
$$


Since $x \geq 0$, we have $\operatorname{Tr}\left(x q_{+}\right)=\operatorname{Tr}\left(q_{+} x q_{+}\right) \geq 0$; hence we have proved that $\alpha+\beta \geq 0$. Likewise, $\alpha-\beta \geq 0$ and we deduce that $P(x) \geq 0$. The argument works as well if $\beta=0$.

We have shown that the map $P: \mathcal{C}_{N} \rightarrow C_{N}$ is positive. Since it is unital, it is a contraction (see e.g. [16, Cor. 2.9]). Since $P$ is selfadjoint, we obtain for free that $P: L^{1}\left(\mathcal{C}_{N}\right) \rightarrow L^{1}\left(\mathcal{C}_{N}\right)$ also is a contraction. We deduce by interpolation that $P: L^{p}\left(\mathcal{C}_{N}\right) \rightarrow L^{p}\left(\mathcal{C}_{N}\right)$ is a contraction for any $1<p<\infty$. Indeed, $L^{p}\left(\mathcal{C}_{N}\right)=$ $\left[\mathcal{C}_{N}, L^{1}\left(\mathcal{C}_{N}\right)\right]_{\frac{1}{p}}$, where [, $]_{\theta}$ denotes the complex interpolation method; see e.g. [19] for details.

We shall now discuss several facts depending on the parity of $N$. It is well known that for any integer $n \geq 1$,

$$
\mathcal{C}_{2 n} \simeq M_{2^{n}} \quad * \text {-isomorphically. }
$$

Moreover this identification induces an isometric identification

$$
L^{p}\left(\mathcal{C}_{2 n}\right) \simeq S_{2^{n}}^{p}
$$

for any $p \geq 1$. Indeed if $\pi: M_{2^{n}} \rightarrow \mathcal{C}_{2 n}$ is the canonical $*$-isomorphism, then we have $\operatorname{Tr}(\pi(x))=2^{-n} \operatorname{tr}(x)$ for any $x \in M_{2^{n}}$. This implies by Lemma 5.1 that for any $p \geq 1$,

$$
2^{\frac{n}{p}} \pi: S_{2^{n}}^{p} \longrightarrow L^{p}\left(\mathcal{C}_{2 n}\right) \quad \text { is a complete isometry. }
$$

We now consider the odd case. For any $n \geq 1$, we set

$$
\rho_{n}=\frac{1}{2}\left(1+i^{n} \omega_{1} \cdots \omega_{2 n} \omega_{2 n+1}\right) \in \mathcal{C}_{2 n+1} .
$$

From (5.1) we have $\left(i^{n} \omega_{1} \cdots \omega_{2 n} \omega_{2 n+1}\right)^{2}=1$; hence $\rho_{n}$ is a (nontrivial) selfadjoint projection. Moreover the anticommutation relations imply that

$$
\left(\omega_{1} \cdots \omega_{2 n} \omega_{2 n+1}\right) \omega_{j}=\omega_{j}\left(\omega_{1} \cdots \omega_{2 n} \omega_{2 n+1}\right)
$$

for any $j=1, \ldots, 2 n+1$. Thus $\omega_{1} \cdots \omega_{2 n} \omega_{2 n+1}$ lies in the center of $\mathcal{C}_{2 n+1}$ and $\rho_{n}$ is therefore central. This induces a direct sum decomposition

$$
\mathcal{C}_{2 n+1}=\rho_{n} \mathcal{C}_{2 n+1} \stackrel{\infty}{\oplus}\left(1-\rho_{n}\right) \mathcal{C}_{2 n+1} .
$$

Regarding $\mathcal{C}_{2 n}$ as a subalgebra of $\mathcal{C}_{2 n+1}$ in the obvious way, we have

$$
\rho_{n} \mathcal{C}_{2 n}=\rho_{n} \mathcal{C}_{2 n+1} \text {. }
$$

Indeed note that $\mathcal{C}_{2 n+1}$ is spanned by $\mathcal{C}_{2 n}$ and the set $\left\{\omega_{A} \omega_{2 n+1}: A \in \mathcal{P}_{2 n}\right\}$. Thus to get this equality, it suffices to check that for any $A \in \mathcal{P}_{2 n}$, we have $\rho_{n} \omega_{A} \omega_{2 n+1} \in \rho_{n} \mathcal{C}_{2 n}$. We have

$$
\begin{aligned}
2 \rho_{n} \omega_{A} \omega_{2 n+1} & =\omega_{A} \omega_{2 n+1}+i^{n} \omega_{1} \cdots \omega_{2 n+1} \omega_{A} \omega_{2 n+1} \\
& =\omega_{A} \omega_{2 n+1}+i^{n}(-1)^{|A|} \omega_{1} \cdots \omega_{2 n} \omega_{A} .
\end{aligned}
$$

Let $y=i^{n}(-1)^{|A|} \omega_{1} \cdots \omega_{2 n} \omega_{A}$. Then $y \in \mathcal{C}_{2 n}$ and

$$
\begin{aligned}
i^{n} \omega_{1} \cdots \omega_{2 n+1} y & =(-1)^{n}(-1)^{|A|} \omega_{1} \cdots \omega_{2 n} \omega_{2 n+1} \omega_{1} \cdots \omega_{2 n} \omega_{A} \\
& =(-1)^{n}\left(\omega_{1} \cdots \omega_{2 n}\right)^{2} \omega_{A} \omega_{2 n+1} \\
& =\omega_{A} \omega_{2 n+1}
\end{aligned}
$$

by (5.11). Hence $\rho_{n} \omega_{A} \omega_{2 n+1}=\rho_{n} y$, which proves the result. 
Since $\mathcal{C}_{2 n}$ is simple, the $*$-representation

$$
\pi_{0}: \mathcal{C}_{2 n} \longrightarrow \mathcal{C}_{2 n+1}, \quad x \mapsto \rho_{n} x,
$$

is one-to-one. The equality we just proved shows that its range is equal to $\rho_{n} \mathcal{C}_{2 n+1}$. Likewise, the $*$-representation

$$
\pi_{1}: \mathcal{C}_{2 n} \longrightarrow \mathcal{C}_{2 n+1}, \quad x \mapsto\left(1-\rho_{n}\right) x,
$$

is a $*$-isomorphism from $\mathcal{C}_{2 n}$ onto $\left(1-\rho_{n}\right) \mathcal{C}_{2 n+1}$. Hence the decomposition (5.4) induces $*$-isomorphisms

$$
\mathcal{C}_{2 n+1} \simeq \mathcal{C}_{2 n} \stackrel{\infty}{\oplus} \mathcal{C}_{2 n} \simeq M_{2^{n}} \stackrel{\infty}{\oplus} M_{2^{n}} .
$$

We observe that $\operatorname{Tr}\left(\pi_{0}(x)\right)=\operatorname{Tr}\left(\pi_{1}(x)\right)=\frac{1}{2} \operatorname{Tr}(x)$ for any $x \in \mathcal{C}_{2 n}$. By Lemma 5.1, this implies that for any $p \geq 1$,

$$
2^{\frac{1}{p}} \pi_{0}, 2^{\frac{1}{p}} \pi_{1}: L^{p}\left(\mathcal{C}_{2 n}\right) \longrightarrow L^{p}\left(\mathcal{C}_{2 n+1}\right) \quad \text { are complete isometries. }
$$

This yields canonical isometric identifications

$$
L^{p}\left(\mathcal{C}_{2 n+1}\right) \simeq L^{p}\left(\mathcal{C}_{2 n}\right) \stackrel{p}{\oplus} L^{p}\left(\mathcal{C}_{2 n}\right) \simeq S_{2^{n}}^{p} \stackrel{p}{\oplus} S_{2^{n}}^{p}
$$

We introduce

$$
F_{n}=\operatorname{Span}\left\{1, \omega_{1}, \ldots, \omega_{2 n}, \omega_{1} \cdots \omega_{2 n}\right\} \subset \mathcal{C}_{2 n} .
$$

This operator space is closely related to $E_{2 n+1}$. Indeed owing to the calculation we made to prove (5.5), we have

$$
\rho_{n} \omega_{2 n+1}=\rho_{n}\left(i^{n} \omega_{1} \cdots \omega_{2 n}\right) .
$$

Hence $\pi_{0}\left(F_{n}\right)=\rho_{n} E_{2 n+1}$. Likewise, we have

$$
\left(1-\rho_{n}\right) \omega_{2 n+1}=-\left(1-\rho_{n}\right)\left(i^{n} \omega_{1} \cdots \omega_{2 n}\right),
$$

and $\pi_{1}\left(F_{n}\right)=\left(1-\rho_{n}\right) E_{2 n+1}$. Arguing as in Lemma 5.2, we have the following.

Lemma 5.3. Let $Q: \mathcal{C}_{2 n} \rightarrow \mathcal{C}_{2 n}$ be the orthogonal projection onto $F_{n}$. Then

$$
\left\|Q: L^{p}\left(\mathcal{C}_{2 n}\right) \longrightarrow L^{p}\left(\mathcal{C}_{2 n}\right)\right\|=1
$$

for any $1 \leq p \leq \infty$.

For any $p \geq 1$, we let $E_{N}^{p}$ denote the space $E_{N}$ regarded as a subspace of $L^{p}\left(\mathcal{C}_{N}\right)$. Likewise for any $n \geq 1$ we let $F_{n}^{p}$ denote the space $F_{n}$ regarded as a subspace of $L^{p}\left(\mathcal{C}_{2 n}\right)$. We define a 'transpose map'

$$
\tau: F_{n} \longrightarrow F_{n}
$$

by letting $\tau(1)=1, \tau\left(\omega_{j}\right)=\omega_{j}$ for any $j=1, \ldots, 2 n$, and $\tau\left(\omega_{1} \cdots \omega_{2 n}\right)=$ $-\omega_{1} \cdots \omega_{2 n}$. The following fact will be used later on in this section.

Lemma 5.4. Consider

$$
\theta=\pi_{1} \tau \pi_{0}^{-1}: \rho_{n} E_{2 n+1} \longrightarrow\left(1-\rho_{n}\right) E_{2 n+1} .
$$

Then $\theta\left(\rho_{n}\right)=1-\rho_{n}$ and $\theta\left(\rho_{n} \omega_{j}\right)=\left(1-\rho_{n}\right) \omega_{j}$ for any $j=1, \ldots, 2 n+1$.

Proof. Only the relation $\theta\left(\rho_{n} \omega_{2 n+1}\right)=\left(1-\rho_{n}\right) \omega_{2 n+1}$ needs a proof. This follows from (5.8) and (5.9). 
Remark 5.5. (1) In the case when $n=1$, we have $F_{1}=\mathcal{C}_{2}$. Consider the so-called Pauli matrices defined by

$$
a=\left[\begin{array}{cc}
1 & 0 \\
0 & -1
\end{array}\right], \quad b=\left[\begin{array}{ll}
0 & 1 \\
1 & 0
\end{array}\right] \quad \text { and } \quad c=\left[\begin{array}{cc}
0 & 1 \\
-1 & 0
\end{array}\right] .
$$

Then the $*$-isomorphism $\pi: M_{2} \rightarrow \mathcal{C}_{2}$ yielding (5.2) in the case $n=1$ is defined by $\pi(1)=1, \pi(a)=\omega_{1}, \pi(b)=\omega_{2}$ and $\pi(c)=\omega_{1} \omega_{2}$. Thus $\tau: F_{1} \rightarrow F_{1}$ corresponds to the classical transpose map of $M_{2}$.

(2) The linear mapping $\sigma: \mathcal{C}_{2 n} \rightarrow \mathcal{C}_{2 n}$ taking 1 to 1 and taking $\omega_{A}$ to $(-1)^{|A|+1} \omega_{A}$ for any nonempty set $A \subset\{1, \ldots, 2 n\}$ is an anti-*-isomorphism. Hence $\sigma$ is an isometry. Moreover this mapping is trace preserving; hence for any $p \geq 1$, $\sigma: L^{p}\left(\mathcal{C}_{2 n}\right) \rightarrow L^{p}\left(\mathcal{C}_{2 n}\right)$ also is an isometry. Since $\tau$ is the restriction of $\sigma$ to $F_{n}$ we deduce that $\tau$ is an isometry on $F_{n}^{p}$.

However in general, $\tau: F_{n}^{p} \rightarrow F_{n}^{p}$ is not a complete isometry. Indeed by (1) above and Proposition 3.2, $\tau: F_{1}^{p} \rightarrow F_{1}^{p}$ is not completely contractive unless $p=2$. The question whether $\tau: F_{n}^{p} \rightarrow F_{n}^{p}$ is a complete isometry is a key issue for our understanding of the $F_{n}^{p}$ 's as operator spaces. This will be discussed in detail in Section 7 below.

(3) Let $F_{n}^{\text {op }}$ be the space $F_{n}$ equipped with the opposite operator space structure (see e.g. [18, Section 2.10]). The mapping $\sigma$ is a $*$-homomorphism from $\mathcal{C}_{2 n}$ into the opposite $C^{*}$-algebra $\mathcal{C}_{2 n}^{\mathrm{op}}$, and $F_{n}^{\mathrm{op}} \subset \mathcal{C}_{2 n}^{\mathrm{op}}$ completely isometrically. Hence $\tau$ is a completely isometric isomorphism from $F_{n}$ onto $F_{n}^{\text {op }}$. More explicitly, for any $a_{0}, \ldots, a_{2 n}, a_{2 n+1}$ in a matrix space $M_{k}$, we have

$$
\begin{aligned}
\| a_{0} \otimes 1+\sum_{j=1}^{2 n} a_{j} \otimes \omega_{j}+ & a_{2 n+1} \otimes \omega_{1} \cdots \omega_{2 n} \|_{M_{k}\left(F_{n}^{\mathrm{op}}\right)} \\
& =\left\|a_{0} \otimes 1+\sum_{j=1}^{2 n} a_{j} \otimes \omega_{j}-a_{2 n+1} \otimes \omega_{1} \cdots \omega_{2 n}\right\|_{M_{k}\left(F_{n}\right)} .
\end{aligned}
$$

For any $p \geq 1$, a similar result holds with $F_{n}^{p}$ in the place of $F_{n}$.

Proposition 5.6. Let $N \geq 2$ be an integer and let $P: \mathcal{C}_{N} \rightarrow \mathcal{C}_{N}$ be the orthogonal projection onto $E_{N}$. Then for any $1 \leq p \neq 2 \leq \infty$, we have

$$
\left\|P: L^{p}\left(\mathcal{C}_{N}\right) \longrightarrow L^{p}\left(\mathcal{C}_{N}\right)\right\|_{2}>1 .
$$

Proof. Let $\mathcal{A}=\operatorname{Span}\left\{1, \omega_{1}, \omega_{2}, \omega_{1} \omega_{2}\right\} \subset \mathcal{C}_{N}$ and let $\mathcal{A}^{p}$ be this space regarded as a subspace of $L^{p}\left(\mathcal{C}_{N}\right)$. Then $P$ maps $\mathcal{A}$ onto its subspace $\operatorname{Span}\left\{1, \omega_{1}, \omega_{2}\right\}$. Under the identification given in Remark 5.5 (1), the latter space coincides with the space of symmetric $2 \times 2$ matrices and we deduce that

$$
\left\|P_{\mid \mathcal{A}}: \mathcal{A}^{p} \longrightarrow \mathcal{A}^{p}\right\|_{2}=\left\|P_{s}: S_{2}^{p} \longrightarrow S_{2}^{p}\right\|_{2},
$$

where $P_{s}$ denotes the canonical projection onto $\mathcal{S}_{2}^{p}$. The result therefore follows from Proposition 3.2 .

For any operators $a_{1} \in S^{p}\left(H_{1}\right)$ and $a_{2} \in S^{p}\left(H_{2}\right)$, with $\left(a_{1}, a_{2}\right) \neq(0,0)$, let us consider the following analog of (2.12):

$$
G=\left\{\left(x \otimes a_{1}, \tau(x) \otimes a_{2}\right): x \in F_{n}^{p}\right\} \subset\left(L^{p}\left(\mathcal{C}_{2 n}\right) \stackrel{p}{\otimes} S^{p}\left(H_{1}\right)\right) \stackrel{p}{\oplus}\left(L^{p}\left(\mathcal{C}_{2 n}\right) \stackrel{p}{\otimes} S^{p}\left(H_{2}\right)\right) .
$$


Proposition 5.7. Assume that $1 \leq p \neq 2<\infty$ and that $n \geq 2$. Then the above space $G$ is 1-complemented but is not [2]-1-complemented.

Proof. Let us assume that $\left\|a_{1}\right\|^{p}+\left\|a_{2}\right\|^{p}=1$ and let $t=\left\|a_{1}\right\|^{p}$. Recall the projection $Q$ from Lemma 5.3. We let

$$
R:\left(L^{p}\left(\mathcal{C}_{2 n}\right) \otimes a_{1}\right) \stackrel{p}{\otimes}\left(L^{p}\left(\mathcal{C}_{2 n}\right) \otimes a_{2}\right) \longrightarrow\left(L^{p}\left(\mathcal{C}_{2 n}\right) \otimes a_{1}\right) \stackrel{p}{\otimes}\left(L^{p}\left(\mathcal{C}_{2 n}\right) \otimes a_{2}\right)
$$

be the linear mapping defined by

$R\left(z_{1} \otimes a_{1}, z_{2} \otimes a_{2}\right)=\left(\left(t Q\left(z_{1}\right)+(1-t) \tau Q\left(z_{2}\right)\right) \otimes a_{1},\left(t \tau Q\left(z_{1}\right)+(1-t) Q\left(z_{2}\right)\right) \otimes a_{2}\right)$,

for any $z_{1}, z_{2} \in L^{p}\left(\mathcal{C}_{2 n}\right)$. This definition is an analog of (2.9) . Arguing as in Section 2 and using Lemma 5.3, we obtain that $R$ is a contractive projection.

To show that $G$ is not [2]-1-complemented, it suffices by Remark 2.9 to show that the above mapping $R$ is not [2]-contractive. The proof is similar to the one of Proposition 5.6. Again we consider $\mathcal{A}=\operatorname{Span}\left\{1, \omega_{1}, \omega_{2}, \omega_{1} \omega_{2}\right\}$ and we note that since $n \geq 2, \tau Q(z)=Q(z)$ for any $z \in \mathcal{A}$. Thus

$$
R\left(z \otimes a_{1}, z \otimes a_{2}\right)=\left(Q(z) \otimes a_{1}, Q(z) \otimes a_{2}\right), \quad z \in \mathcal{A} .
$$

Consequently,

$$
\|R\|_{2} \geq\left\|Q: \mathcal{A}^{p} \longrightarrow \mathcal{A}^{p}\right\|_{2}=\left\|P_{s}: S_{2}^{p} \longrightarrow S_{2}^{p}\right\|_{2},
$$

and the latter norm is $>1$ by Proposition 3.2 .

Remark 5.8. (1) As a special case $\left(a_{1}=a, a_{2}=0\right)$, we obtain that for any $n \geq 2$ and any nonzero $a \in S^{p}(H)$, the space

$$
F_{n} \otimes a \subset L^{p}\left(\mathcal{C}_{2 n}\right) \stackrel{p}{\otimes} S^{p}(H)
$$

is not [2]-1-complemented for $p \neq 2$. Equivalently,

$$
\left\|Q: L^{p}\left(\mathcal{C}_{N}\right) \longrightarrow L^{p}\left(\mathcal{C}_{N}\right)\right\|_{2}>1
$$

whenever $p \neq 2$.

(2) It follows from Proposition 5.6 that for any $N \geq 2$ and nonzero $a \in S^{p}(H)$, the space

$$
E_{N} \otimes a \subset L^{p}\left(\mathcal{C}_{N}\right) \stackrel{p}{\otimes} S^{p}(H)
$$

is not [2]-1-complemented for $p \neq 2$.

Our next goal is to prove Theorem 5.11 below. We need more information on spin systems. We noticed in Section 2 that for any $N \geq 1$, the Fermions $\left(\omega_{1}, \ldots, \omega_{N}\right)$ form a spin system. Also it follows from (5.1) that for any $n \geq 1$, the $(2 n+1)$-tuple $\left(\omega_{1}, \ldots, \omega_{2 n}, i^{n} \omega_{1} \cdots \omega_{2 n}\right)$ is a spin system. The next lemma shows that these are essentially the only examples.

Lemma 5.9. Let $n \geq 1$ be any integer.

(1) Let $\left(s_{1}, \ldots, s_{2 n}\right)$ be a spin system with an even cardinal. There is a (necessarily unique) $*$-isomorphism

$$
\pi: \mathcal{C}_{2 n} \longrightarrow C^{*}\left\langle s_{1}, \ldots, s_{2 n}\right\rangle
$$

such that $\pi\left(\omega_{j}\right)=s_{j}$ for any $j=1, \ldots, 2 n$. 
(2) Let $\left(s_{1}, \ldots, s_{2 n+1}\right)$ be a spin system with an odd cardinal and let

$$
q=\frac{1}{2}\left(1+i^{n} s_{1} \cdots s_{2 n} s_{2 n+1}\right) .
$$

If $q \notin\{0,1\}$, then there is a (necessarily unique) *-isomorphism

$$
\pi: \mathcal{C}_{2 n+1} \longrightarrow C^{*}\left\langle s_{1}, \ldots, s_{2 n+1}\right\rangle
$$

such that $\pi\left(\omega_{j}\right)=s_{j}$ for any $j=1, \ldots, 2 n+1$. In this case, we have $\pi\left(\rho_{n}\right)=q$.

Note that in (2) above, $q=0$ if and only if $s_{2 n+1}=-i^{n} s_{1} \cdots s_{2 n}$. In this case (1) ensures that there is a $*$-isomorphism $\pi: \mathcal{C}_{2 n} \rightarrow C^{*}\left\langle s_{1}, \ldots, s_{2 n}\right\rangle$ such that $\pi\left(\omega_{j}\right)=s_{j}$ for any $j \leq 2 n$ and $\pi\left(i^{n} \omega_{1} \cdots \omega_{2 n}\right)=s_{2 n+1}$. A similar comment applies when $q=1$.

For simplicity, we now let $c_{j}$ (instead of $c_{n, j}$ ) denote the creation operators on $\Lambda_{n}$ and recall that we defined $\omega_{j}=c_{j}+c_{j}^{*}$ for any $j=1, \ldots, n$. Next we let

$$
\omega_{-j}=\frac{c_{j}-c_{j}^{*}}{i} \quad j=1, \ldots, n .
$$

It is well known (and easy to check) that the $2 n$-tuple $\left(\omega_{1}, \ldots, \omega_{n}, \omega_{-1}, \ldots, \omega_{-n}\right)$ is a spin system.

Proof of Lemma 5.9. Let $\left(s_{1}, \ldots, s_{2 n}\right)$ be an arbitrary spin system and set

$$
v_{j}=\frac{s_{j}+i s_{n+j}}{2}, \quad j=1, \ldots, n .
$$

These operators satisfy the so-called canonical anticommutation relations (CAR); that is,

$$
v_{i} v_{j}^{*}+v_{j}^{*} v_{i}=\delta_{i, j} \quad \text { and } \quad v_{i} v_{j}+v_{j} v_{i}=0
$$

for any $1 \leq i, j \leq n$. The creation operators $c_{1}, \ldots, c_{n}$ satisfy the CAR as well; hence according to e.g. [4, p. 15], there is a *-isomorphism $\pi: C^{*}\left\langle c_{1}, \ldots, c_{n}\right\rangle \rightarrow$ $C^{*}\left\langle v_{1}, \ldots, v_{n}\right\rangle$ such that $\pi\left(c_{j}\right)=v_{j}$ for all $j$. Equivalently,

$$
\pi: C^{*}\left\langle\omega_{1}, \ldots, \omega_{n}, \omega_{-1}, \ldots, \omega_{-n}\right\rangle \longrightarrow C^{*}\left\langle s_{1}, \ldots, s_{2 n}\right\rangle
$$

is a $*$-isomorphism which satisfies $\pi\left(\omega_{j}\right)=s_{j}$ and $\pi\left(\omega_{-j}\right)=s_{n+j}$ for any $j=$ $1, \ldots, n$. The assertion (1) follows at once.

Now let $\left(s_{1}, \ldots, s_{2 n}, s_{2 n+1}\right)$ be a spin system with an odd cardinal, and let $\omega_{1}, \ldots, \omega_{2 n}, \omega_{2 n+1}$ be the usual Fermions. Suppose that $q \notin\{0,1\}$. Then we can mimic what we did before with Fermions and we obtain that $q$ is a central projection of $C^{*}\left\langle s_{1}, \ldots, s_{2 n+1}\right\rangle$, and that we have

$$
\begin{aligned}
& C^{*}\left\langle s_{1}, \ldots, s_{2 n+1}\right\rangle=q C^{*}\left\langle s_{1}, \ldots, s_{2 n+1}\right\rangle \stackrel{\infty}{\oplus}(1-q) C^{*}\left\langle s_{1}, \ldots, s_{2 n+1}\right\rangle \\
& \simeq C^{*}\left\langle s_{1}, \ldots, s_{2 n}\right\rangle \stackrel{\infty}{\oplus} C^{*}\left\langle s_{1}, \ldots, s_{2 n}\right\rangle \text {. }
\end{aligned}
$$

Then using the $*$-isomorphism $\mathcal{C}_{2 n} \rightarrow C^{*}\left\langle s_{1}, \ldots, s_{2 n}\right\rangle$ given by (1), we deduce the desired $*$-isomorphism from $\mathcal{C}_{2 n+1}$ onto $C^{*}\left\langle s_{1}, \ldots, s_{2 n+1}\right\rangle$.

As in Section 2, we let $P_{n}: \Lambda_{n} \rightarrow \Lambda_{n}$ be the orthogonal projection onto the space generated by tensor products of even rank.

Lemma 5.10. With the notation introduced before Lemma [5.9, we have

$$
P_{n}=\frac{1}{2}\left(1+i^{n} \omega_{n} \omega_{-n} \cdots \omega_{1} \omega_{-1}\right) .
$$


Proof. Let $A \subset\{1, \ldots, n\}$ and recall (2.10). For any $j=1, \ldots, n$, we have

$$
\omega_{j} \omega_{-j}=-i\left(c_{j}+c_{j}^{*}\right)\left(c_{j}-c_{j}^{*}\right)=i\left(c_{j} c_{j}^{*}-c_{j}^{*} c_{j}\right) .
$$

Hence $\omega_{j} \omega_{-j}\left(e_{A}\right)=i e_{A}$ if $j \in A$, and $\omega_{j} \omega_{-j}\left(e_{A}\right)=-i e_{A}$ if $j \notin A$. Consequently

$$
\omega_{n} \omega_{-n} \cdots \omega_{1} \omega_{-1}\left(e_{A}\right)=(-1)^{n-|A|} i^{n} e_{A},
$$

which implies the result.

Theorem 5.11. Let $X \subset S^{p}(\mathcal{H}, \mathcal{K})$ with $\operatorname{dim}(X) \geq 5$ and $1 \leq p \neq 2<\infty$. If $X$ is a spinorial space, then $X$ is not [2]-1-complemented.

Proof. We first consider spinorial spaces with an even dimension. Let $n \geq 3$ be an integer. By Lemma 2.3 and Definition 2.7 it suffices to show that the space $Z$ given by (2.12) is not [2]-1-complemented.

We need some preliminary observations concerning $A H_{n}$ and $B H_{n}$, which will lead to a formal relationship between $Z$ and the space $G$ given by (5.10), with $(n-1)$ instead of $n$. Using the notation (5.11), we have

$$
A H_{n}=\operatorname{Span}\left\{\omega_{j} P_{n}, \omega_{-j} P_{n}: 1 \leq j \leq n\right\} .
$$

For any $j \in\{-n, \ldots,-1\} \cup\{1, \ldots, n-1\}$ we let $w_{j}^{\prime}=i \omega_{n} \omega_{j}$. Then $\omega_{j}=i \omega_{j}^{\prime} \omega_{n}$ and for any $j=1, \ldots, n-1$, we have $\omega_{j} \omega_{-j}=\omega_{j}^{\prime} \omega_{-j}^{\prime}$. Applying Lemma [5.10, this yields

$$
P_{n}=\frac{1}{2}\left(1+i^{n-1} \omega_{-n}^{\prime} \omega_{n-1}^{\prime} \omega_{-(n-1)}^{\prime} \cdots \omega_{1}^{\prime} \omega_{-1}^{\prime}\right) .
$$

Let $W: B\left(\Lambda_{n}\right) \rightarrow B\left(\Lambda_{n}\right)$ be the left multiplication by $i \omega_{n}$. Later on we will use the obvious fact that

$$
W: S^{p}\left(\Lambda_{n}\right) \longrightarrow S^{p}\left(\Lambda_{n}\right) \quad \text { is a complete isometry. }
$$

According to the above expression of $P_{n}$, the action of $W$ on $A H_{n}$ is given by

$$
W\left(\omega_{j} P_{n}\right)=\omega_{j}^{\prime}\left(\frac{1+i^{n-1} \omega_{-n}^{\prime} \omega_{n-1}^{\prime} \cdots \omega_{1}^{\prime} \omega_{-1}^{\prime}}{2}\right)
$$

if $j$ belongs to $\{-n, \ldots,-1\} \cup\{1, \ldots, n-1\}$, and

$$
W\left(\omega_{n} P_{n}\right)=i\left(\frac{1+i^{n-1} \omega_{-n}^{\prime} \omega_{n-1}^{\prime} \cdots \omega_{1}^{\prime} \omega_{-1}^{\prime}}{2}\right) .
$$

It is easy to check that the $(2 n-1)$-tuple $\left(\omega_{-n}^{\prime}, \omega_{n-1}^{\prime}, \omega_{-(n-1)}^{\prime}, \ldots, \omega_{1}^{\prime}, \omega_{-1}^{\prime}\right)$ is a spin system. Moreover the product of these spins is

$$
\omega_{-n}^{\prime} \omega_{n-1}^{\prime} \omega_{-(n-1)}^{\prime} \cdots \omega_{1}^{\prime} \omega_{-1}^{\prime}=i \omega_{n} \omega_{-n} \omega_{n-1} \omega_{-(n-1)} \cdots \omega_{1} \omega_{-1},
$$

which is not a multiple of 1 . Thus by Lemma 5.9 (2), there is a faithful *representation $\pi: \mathcal{C}_{2 n-1} \rightarrow B\left(\Lambda_{n}\right)$ such that

$$
\pi\left(\omega_{1}\right)=\omega_{-n}^{\prime}, \pi\left(\omega_{2}\right)=\omega_{n-1}^{\prime}, \ldots, \pi\left(\omega_{2 n-2}\right)=\omega_{1}^{\prime}, \pi\left(\omega_{2 n-1}\right)=\omega_{-1}^{\prime} .
$$

Furthermore, $\operatorname{tr}\left(\pi\left(\omega_{A}\right)\right)=0$ for any $A \in \mathcal{P}_{2 n-1} \backslash\{\emptyset\}$. Thus $\operatorname{tr}(\pi(x))=2^{n} \operatorname{Tr}(x)$ for any $x \in \mathcal{C}_{2 n-1}$ and we deduce (by Lemma 5.1) that

$$
2^{-\frac{n}{p}} \pi: L^{p}\left(\mathcal{C}_{2 n-1}\right) \longrightarrow S^{p}\left(\Lambda_{n}\right) \text { is a complete isometry. }
$$

Since $\pi$ is multiplicative, we see that $\pi\left(\rho_{n-1}\right)=P_{n}$ by comparing (5.3) and (5.12). Consequently, we have

$$
\pi\left(\rho_{n-1} E_{2 n-1}\right)=W\left(A H_{n}\right) .
$$


Also we have

$$
\pi\left(\left(1-\rho_{n-1}\right) E_{2 n-1}\right)=W\left(B H_{n}\right),
$$

and a thorough look at (5.14) and (5.15) actually shows that

$$
\pi^{-1} W \kappa W^{-1} \pi: \rho_{n-1} E_{2 n-1} \longrightarrow\left(1-\rho_{n-1}\right) E_{2 n-1}
$$

is nothing but the mapping which takes $\rho_{n-1} y$ to $\left(1-\rho_{n-1}\right) y$ for any $y \in E_{2 n-1}$.

As before we let $\pi_{0}, \pi_{1}: \mathcal{C}_{2 n-2} \rightarrow \mathcal{C}_{2 n-1}$ be the left multiplications by $\rho_{n-1}$ and $\left(1-\rho_{n-1}\right)$, respectively. Then Lemma [5.4, with $(n-1)$ instead of $n$, yields the relation

$$
\tau=\left(\pi_{1}^{-1} \pi^{-1} W\right) \circ \kappa \circ\left(W^{-1} \pi \pi_{0}\right): F_{n-1} \longrightarrow F_{n-1} .
$$

Set $\Gamma_{i}=W^{-1} \pi \pi_{i}: \mathcal{C}_{2 n-2} \rightarrow B\left(\Lambda_{n}\right)$, for $i=1,2$. It follows from (5.7), (5.13) and (5.16) that

$$
2^{-\frac{(n-1)}{p}} \Gamma_{0}, 2^{-\frac{(n-1)}{p}} \Gamma_{1}: L^{p}\left(\mathcal{C}_{2 n-2}\right) \longrightarrow S^{p}\left(\Lambda_{n}\right) \quad \text { are complete isometries. }
$$

Let us now assume that $Z$ is [2]-1-complemented in $S^{p}\left(\Lambda_{n} \stackrel{2}{\otimes} H_{1}\right) \stackrel{p}{\oplus} S^{p}\left(\Lambda_{n} \stackrel{2}{\otimes} H_{2}\right)$. Then by (5.18), the space

$$
\begin{aligned}
\left\{\left(\Gamma_{0}^{-1}(x) \otimes a_{1}, \Gamma_{1}^{-1} \kappa(x) \otimes a_{2}\right)\right. & \left.: x \in A H_{n}^{p}\right\} \\
& \subset\left(L^{p}\left(\mathcal{C}_{2 n-2}\right) \stackrel{p}{\otimes} S^{p}\left(H_{1}\right)\right) \stackrel{p}{\oplus}\left(L^{p}\left(\mathcal{C}_{2 n-2}\right) \stackrel{p}{\otimes} S^{p}\left(H_{2}\right)\right)
\end{aligned}
$$

is [2]-1-complemented as well. According to (5.17), this space coincides with the space $G$ of (5.10). By Proposition [5.7, we obtain a contradiction.

The proof for the spinorial spaces of odd dimension is similar, using Remark 5.8 (2) in the place of Proposition 5.7. We skip the details.

Remark 5.12. It follows from Proposition 5.7 its subsequent remark and the proof of Theorem 5.11 that any spinorial space is 1-complemented. Also it follows from Remark 5.5 (1) and the proof of Theorem 5.11 that the exchange map $\kappa$ defined by (2.11) is an isometry on $A H_{n}^{p}$ for any $p$.

\section{MAin Results}

In this section we state our main results and prove Theorem 1.1, mostly by combining results proved in the last three sections. Throughout we assume that $1 \leq p \neq 2<\infty$.

Theorem 6.1. Let $\mathcal{H}, \mathcal{K}$ be Hilbert spaces and let $X \subset S^{p}(\mathcal{H}, \mathcal{K})$ be an indecomposable subspace. The following are equivalent.

(i) $X$ is completely 1 -complemented in $S^{p}(\mathcal{H}, \mathcal{K})$.

(ii) $X$ is [2]-1-complemented in $S^{p}(\mathcal{H}, \mathcal{K})$.

(iii) There exist index sets $I, J$ and an operator $a \in S^{p}(H)$ such that

$$
X \sim S_{I, J}^{p} \otimes a .
$$

Proof. The implication '(i) $\Rightarrow$ (ii)' is obvious, and '(iii) $\Rightarrow$ (i)' follows from Lemma 2.3 To prove the hard implication '(ii) $\Rightarrow$ (iii)', assume that $X$ is [2]-1-complemented in $S^{p}(\mathcal{H}, \mathcal{K})$ and that $\operatorname{dim}(X)>1$. By Proposition 3.2 and Theorem 5.11, $X$ is neither a spinorial space of dimension $\geq 5$ nor a space of symmetric or anti-symmetric matrices. Hence according to the Arazy-Friedman Theorem 2.8, $X$ is either a space of rectangular matrices or is equivalent to a finite dimensional Hilbert space of 
the form (2.14). In the latter case, Proposition 4.2 ensures that $X$ is actually equivalent to a space of rectangular matrices. Then Proposition 3.1 finally shows that $X$ satisfies (iii).

Proposition 6.2. Let $\left(I_{\alpha}\right)_{\alpha}$ and $\left(J_{\alpha}\right)_{\alpha}$ be two families of indices, and let

$$
u: \underset{\alpha}{p} S_{I_{\alpha}, J_{\alpha}}^{p} \longrightarrow S^{p}(\mathcal{H}, \mathcal{K})
$$

be a complete isometry. Then the range of $u$ is completely 1-complemented.

Proof. We may assume that $\mathcal{H}=\mathcal{K}$. First consider the case when the family is a singleton; that is, we have index sets $I, J$ and a complete isometry $u: S_{I, J}^{p} \rightarrow S^{p}(\mathcal{H})$, and we wish to show that its range is completely 1-complemented. In the "square case', that is, $I=J$, this is a special case of [9, Prop. 3.3]. In fact it can also be quickly deduced from [1, Thm. 2.1]. More generally, it is not hard to deduce the result from the latter reference if $I \geq 2$ and $J \geq 2$. The sequel of the proof is necessary only to treat the case when $I$ or $J$ is equal to 1 , although we will write it for general $I, J$. We will show how to reduce to the 'square case'.

We may consider a complete isometry $v: S_{J, I}^{p} \rightarrow S^{p}(\mathcal{H})$. For example the mapping $v$ defined by $v(x)={ }^{t}\left[u\left({ }^{t} x\right)\right]$ for any $x \in S_{J, I}^{p}$ is a complete isometry (here ${ }^{~}{ }^{t}$, stands for the transposition). Recall that

$$
S_{I \times J}^{p} \simeq S_{I, J}^{p} \stackrel{p}{\otimes} S_{J, I}^{p} \simeq S_{J, I}^{p} \stackrel{p}{\otimes} S_{I, J}^{p} .
$$

Hence the tensor map $u \otimes v$ extends to a complete isometry

$$
u \bar{\otimes} v: S_{I \times J}^{p} \longrightarrow S^{p}(\mathcal{H}) \stackrel{p}{\otimes} S^{p}(\mathcal{H}) \simeq S^{p}(\mathcal{H} \stackrel{2}{\otimes} \mathcal{H}) .
$$

We know from the above discussion that the range of $u \bar{\otimes} v$ is completely 1-complemented. Thus there exists a completely contractive mapping

$$
w: S^{p}(\mathcal{H}) \stackrel{p}{\otimes} S^{p}(\mathcal{H}) \longrightarrow S_{I, J}^{p} \stackrel{p}{\otimes} S_{J, I}^{p}
$$

such that $Q \circ u \bar{\otimes} v$ is the identity of $S_{I, J}^{p} \stackrel{p}{\otimes} S_{J, I}^{p}$. Let $z \in S_{J, I}^{p}$ and $z^{*} \in\left(S_{J, I}^{p}\right)^{*}$ such that $\left\langle z^{*}, z\right\rangle=\|z\|=\left\|z^{*}\right\|=1$. By e.g. [5, Cor. 2.2.3], $z^{*}$ is a complete contraction on $S_{J, I}^{p}$; hence $I d \otimes z^{*}: S_{I, J}^{p} \otimes S_{J, I}^{p} \rightarrow S_{I, J}^{p}$ extends to a complete contraction

$$
I d \bar{\otimes} z^{*}: S_{I, J}^{p} \stackrel{p}{\otimes} S_{J, I}^{p} \longrightarrow S_{I, J}^{p} .
$$

Let $\widetilde{w}: S^{p}(\mathcal{H}) \rightarrow S_{I, J}^{p}$ be defined by

$$
\widetilde{w}(y)=\left[\left(I d \bar{\otimes} z^{*}\right) \circ w\right](y \otimes v(z)), \quad y \in S^{p}(\mathcal{H}) .
$$

This is a completely contractive map and for any $x \in S_{I, J}^{p}$, we have

$$
\widetilde{w} \circ u(x)=\left[\left(I d \otimes z^{*}\right) \circ w\right](u(x) \otimes v(z))=I d \otimes z^{*}(x \otimes z)=x .
$$

Thus $\widetilde{w} \circ u$ is the identity of $S_{I, J}^{p}$, which proves the result.

We now consider the general case and we will apply results on orthogonality reviewed in Section 2. Let $u$ be as in the proposition and for any $\alpha$, let $X_{\alpha}=$ $u\left(S_{I_{\alpha}, J_{\alpha}}^{p}\right) \subset S^{p}(\mathcal{H}, \mathcal{K})$. Since $u$ is an isometry, it follows from (2.5) that the $X_{\alpha}$ 's are pairwise orthogonal. Thus there exist pairwise orthogonal closed subspaces $H_{\alpha} \subset \mathcal{H}$ as well as pairwise orthogonal closed subspaces $K_{\alpha} \subset \mathcal{H}$ such that $X_{\alpha} \subset$ 
$S^{p}\left(H_{\alpha}, K_{\alpha}\right)$. It follows from the first part of this proof that for any $\alpha$, there is a completely contractive projection

$$
P_{\alpha}: S^{p}\left(H_{\alpha}, K_{\alpha}\right) \longrightarrow S^{p}\left(H_{\alpha}, K_{\alpha}\right)
$$

whose range equals $X_{\alpha}$. Moreover the $p$-direct sum $\underset{\alpha}{\oplus} S^{p}\left(H_{\alpha}, K_{\alpha}\right) \subset S^{p}(\mathcal{H}, \mathcal{K})$ is the range of a completely contractive projection $Q: S^{p}(\mathcal{H}, \mathcal{K}) \rightarrow S^{p}(\mathcal{H}, \mathcal{K})$. We can now define a mapping $P: S^{p}(\mathcal{H}, \mathcal{K}) \rightarrow S^{p}(\mathcal{H}, \mathcal{K})$ by letting

$$
P(z)=\left(P_{\alpha}(Q(z))\right)_{\alpha}, \quad z \in S^{p}(\mathcal{H}, \mathcal{K}) .
$$

Clearly $P$ is a completely contractive projection whose range is equal to the range of $u$.

Proof of Theorem 1.1. The implication '(iii) $\Rightarrow$ (iv)' follows from Lemma 2.3, '(iv) $\Rightarrow(\mathrm{i})^{\prime}$ ' is given by Proposition 6.2 and '(i) $\Rightarrow$ (ii)' is obvious. Now assume (ii). By [3. Prop. 2.2], $X$ can be written as the $p$-direct sum $X=\bigoplus_{\alpha}^{p} X_{\alpha}$ of pairwise orthogonal indecomposable subspaces. Then it is plain that each ${ }^{\alpha}{ }_{\alpha}$ is [2]-1-complemented as well. Applying Theorem 6.1 and an obvious direct sum argument, we deduce that (iii) holds true.

It follows from Theorem 1.1 that if $X \subset S^{p}(\mathcal{H}, \mathcal{K})$ and $Y \subset S^{p}\left(\mathcal{H}^{\prime}, \mathcal{K}^{\prime}\right)$ are completely isometric, then $X$ is completely 1-complemented if and only if $Y$ is completely 1-complemented.

Remark 6.3. Let $X \subset B(\mathcal{H}, \mathcal{K})$ be a $w^{*}$-closed subspace. Using Theorem 1.1 for $p=1$ and an elementary duality argument, we find that if $X$ is the range of a $w^{*}$-continuous completely contractive projection $B(\mathcal{H}, \mathcal{K}) \rightarrow B(\mathcal{H}, \mathcal{K})$, then there exist two families of Hilbert spaces $\left(H_{\alpha}\right)_{\alpha}$ and $\left(K_{\alpha}\right)_{\alpha}$ such that $X$ is completely isometrically and $w^{*}$-homeomorphically isomorphic to $\stackrel{\infty}{\oplus} B\left(H_{\alpha}, K_{\alpha}\right)$.

The converse does not hold true. Indeed there is an example in [6, Section 3] of a $w^{*}$-continuous complete isometry $u: B(H) \rightarrow B(\mathcal{H})$ whose range cannot be the range of a $w^{*}$-continuous completely contractive projection $B(\mathcal{H}) \rightarrow B(\mathcal{H})$.

For the sake of completeness, we note the following related result going back to 21: A von Neumann algebra $M \subset B(\mathcal{H})$ is the range of a $w^{*}$-continuous contractive projection if and only if it can be written as

$$
M \simeq \underset{\alpha}{\infty} B\left(H_{\alpha}\right)
$$

where ' $\simeq$ ' indicates a von Neumann algebra identification. It turns out that the same result holds true without the word 'contractive'; see [10, Remark 4.7].

\section{TRANSPOSE MAP ON THE SPIN FACTOR}

We recall that for any integer $n \geq 1$, the transpose map $\tau: F_{n} \rightarrow F_{n}$ is the linear isometry defined by

$\tau(1)=1, \quad \tau\left(\omega_{j}\right)=\omega_{j} \quad$ for any $j=1, \ldots, 2 n, \quad$ and $\quad \tau\left(\omega_{1} \cdots \omega_{2 n}\right)=-\omega_{1} \cdots \omega_{2 n}$.

In this section we consider the question whether $\tau$ is a complete contraction (equivalently, a complete isometry) on $F_{n}^{p}$ for $1 \leq p<\infty$, or on $F_{n}=F_{n}^{\infty} \subset \mathcal{C}_{2 n}$, and we give applications. 
In the sequel we use the notation

$$
s_{0}=1, \quad s_{j}=\omega_{j} \quad \text { for any } j=1, \ldots, 2 n, \quad \text { and } \quad s_{2 n+1}=\omega_{1} \cdots \omega_{2 n} .
$$

For any $(2 n+2)$-tuple of signs $\Theta=\left(\theta_{0}, \theta_{1}, \ldots, \theta_{2 n+1}\right) \in\{-1,1\}^{2 n+2}$, one can more generally consider the map $\tau_{\Theta}: F_{n} \rightarrow F_{n}$ defined by

$$
\tau_{\Theta}\left(s_{j}\right)=\theta_{j} s_{j} \quad \text { for any } j=0, \ldots, 2 n+1 .
$$

The completely bounded norm of this map only depends on the parity of the number of minus signs in the sequence $\Theta$. Indeed let $\pi: \mathcal{C}_{2 n} \rightarrow \mathcal{C}_{2 n}$ be the $*$-isomorphism taking $\omega_{j}$ to $\theta_{0} \theta_{j} \omega_{j}$ for any $j=1, \ldots, 2 n$ and recall that $\pi: L^{p}\left(\mathcal{C}_{2 n}\right) \rightarrow L^{p}\left(\mathcal{C}_{2 n}\right)$ is a complete isometry for any $1 \leq p \leq \infty$. Then $\theta_{0} \tau_{\Theta}$ is equal to the restriction of $\pi$ to $F_{n}$ if $\theta_{0} \theta_{1} \cdots \theta_{2 n+1}=1$ and is equal to $\tau \circ \pi_{\mid F_{n}}$ if $\theta_{0} \theta_{1} \cdots \theta_{2 n+1}=-1$. Hence for any $1 \leq p \leq \infty, \tau_{\Theta}: F_{n}^{p} \rightarrow F_{n}^{p}$ is a complete isometry if $\theta_{0} \theta_{1} \cdots \theta_{2 n+1}=1$ whereas $\left\|\tau_{\Theta}: F_{n}^{p} \rightarrow F_{n}^{p}\right\|_{c b}=\left\|\tau: F_{n}^{p} \rightarrow F_{n}^{p}\right\|_{c b}$ if $\theta_{0} \theta_{1} \cdots \theta_{2 n+1}=-1$.

We start with a precise estimate in the case $p=\infty$. Later on we will find the same estimate for $p=1$.

Proposition 7.1. For any $n \geq 1$, we have

$$
\left\|\tau: F_{n} \longrightarrow F_{n}\right\|_{c b}=\frac{n+1}{n} .
$$

Proof. For any $j=0,1, \ldots, 2 n+1$, we let $\pi_{j}: \mathcal{C}_{2 n} \rightarrow \mathcal{C}_{2 n}$ be the $*$-representation defined by letting $\pi_{j}(x)=s_{j}^{*} x s_{j}$ for any $x \in \mathcal{C}_{2 n}$. Of course, $\pi_{0}$ is just the identity map. It is easy to check that for any set $A \in \mathcal{P}_{2 n}$ and for any $1 \leq j \leq 2 n$, we have

$$
\begin{aligned}
\pi_{j}\left(\omega_{A}\right)=\omega_{j} \omega_{A} \omega_{j} & =\omega_{A} & & \text { if }|A| \text { is even and } j \notin A ; \\
& =-\omega_{A} & & \text { if }|A| \text { is even and } j \in A ; \\
& =-\omega_{A} & & \text { if }|A| \text { is odd and } j \notin A ; \\
& =\omega_{A} & & \text { if }|A| \text { is odd and } j \in A .
\end{aligned}
$$

Then we have

$$
\pi_{2 n+1}\left(\omega_{A}\right)=\omega_{2 n} \cdots \omega_{1} \omega_{A} \omega_{1} \cdots \omega_{2 n}=(-1)^{|A|} \omega_{A} .
$$

It follows from these computations that for the $(2 n+2)$-tuple $\Theta=(-1,1, \ldots, 1)$ we have

$$
2 n \tau_{\Theta}=\pi_{0}-\sum_{j=1}^{2 n+1} \pi_{j}
$$

Hence according to the discussion above this proposition, we have

$$
\left\|\tau: F_{n} \longrightarrow F_{n}\right\|_{c b}=\frac{1}{2 n}\left\|\pi_{0}-\sum_{j=1}^{2 n+1} \pi_{j}: F_{n} \longrightarrow F_{n}\right\|_{c b} .
$$

This yields the above estimate $\|\tau\|_{c b} \leq(n+1) / n$.

We now turn to the lower estimate. By the definition of $\tau$, we have

$$
\begin{aligned}
\| \sum_{j=0}^{2 n} s_{j} \otimes s_{j}+s_{2 n+1}^{*} \otimes s_{2 n+1} & \|_{\mathcal{C}_{2 n} \otimes \min } \mathcal{C}_{2 n} \\
\leq\|\tau\| & \left\|\sum_{j=0}^{2 n} s_{j} \otimes s_{j}-s_{2 n+1}^{*} \otimes s_{2 n+1}\right\|_{\mathcal{C}_{2 n} \otimes \min } \mathcal{C}_{2 n}
\end{aligned}
$$


where $\otimes_{\min }$ stands for the minimal (or spatial) tensor product of $C^{*}$-algebras. Since $\mathcal{C}_{2 n} \simeq M_{2^{n}}$ is a matrix space, the bilinear map $\mathcal{C}_{2 n} \times \mathcal{C}_{2 n} \rightarrow B\left(L^{2}\left(\mathcal{C}_{2 n}\right)\right)$ taking any $(a, b)$ to the mapping $T \rightarrow a T b$ (for $a, b \in \mathcal{C}_{2 n}$ and $T \in L^{2}\left(\mathcal{C}_{2 n}\right)$ ) extends to an isometric isomorphism

$$
\mathcal{C}_{2 n} \otimes_{\min } \mathcal{C}_{2 n} \simeq B\left(L^{2}\left(\mathcal{C}_{2 n}\right)\right)
$$

In this identification, $s_{j}^{*} \otimes s_{j}$ corresponds to $\pi_{j}$ for any $j=0, \ldots, 2 n+1$. Furthermore, it follows from the first part of this proof that each $\pi_{j}$ is a diagonal operator with respect to the orthonormal basis $\left(\omega_{A}\right)_{A \in \mathcal{P}_{2 n}}$, whose eigenvalues are either +1 or -1 . Moreover if $A \in \mathcal{P}_{2 n}$ is such that $\pi_{j}\left(\omega_{A}\right)=\omega_{A}$ for any $1 \leq j \leq 2 n$, then $A=\emptyset$. We deduce that the eigenvalues of the diagonal operator $\pi_{0}+\cdots+\pi_{2 n}-\pi_{2 n+1}$ are integers belonging to $[-2 n, 2 n]$. Thus

$$
\left\|\sum_{j=0}^{2 n} s_{j} \otimes s_{j}-s_{2 n+1}^{*} \otimes s_{2 n+1}\right\|_{\mathcal{C}_{2 n} \otimes \min \mathcal{C}_{2 n}}=\left\|\sum_{j=0}^{2 n} \pi_{j}-\pi_{2 n+1}\right\|_{L^{2} \rightarrow L^{2}} \leq 2 n .
$$

On the other hand, $\pi_{j}\left(\omega_{\emptyset}\right)=1$ for any $j=0, \ldots, 2 n+1$; hence

$$
\left\|\sum_{j=0}^{2 n} s_{j} \otimes s_{j}+s_{2 n+1}^{*} \otimes s_{2 n+1}\right\|_{\mathcal{C}_{2 n} \otimes \min } \mathcal{C}_{2 n}=\left\|\sum_{j=0}^{2 n} \pi_{j}+\pi_{2 n+1}\right\|_{L^{2} \rightarrow L^{2}}=2 n+2 .
$$

Consequently, we have $\|\tau\|_{c b} \geq(n+1) / n$.

Remark 7.2. For a linear map $u: \mathcal{C}_{2 n} \rightarrow \mathcal{C}_{2 n}$, the Wittstock factorization theorem asserts that

$$
\|u\|_{c b}=\inf \left\{\left\|\sum_{j} a_{j}^{*} a_{j}\right\|^{\frac{1}{2}}\left\|\sum_{j} b_{j}^{*} b_{j}\right\|^{\frac{1}{2}}\right\}
$$

where the infimum runs over all finite families $\left(a_{j}\right)_{j}$ and $\left(b_{j}\right)_{j}$ in $\mathcal{C}_{2 n}$ such that

$$
u(x)=\sum_{j} a_{j}^{*} x b_{j}, \quad x \in \mathcal{C}_{2 n} .
$$

(See e.g. [5, Sect. 5.3].) The above proof yields an extension $u: \mathcal{C}_{2 n} \rightarrow \mathcal{C}_{2 n}$ of $\tau: F_{n} \rightarrow F_{n}$, as well as a factorization of the type (7.3) such that

$$
\left\|\sum_{j} a_{j}^{*} a_{j}\right\|=\left\|\sum_{j} b_{j}^{*} b_{j}\right\|=\|\tau\|_{c b} .
$$

Indeed this is obtained by taking

$$
a_{j}=(2 n)^{-\frac{1}{2}} s_{2 n+1} s_{j} \quad \text { for } j=1, \ldots 2 n+1, \quad a_{2 n+2}=(2 n)^{-\frac{1}{2}} s_{2 n+2},
$$

and then $b_{j}=a_{j}$ for $j=1, \ldots, 2 n+1$ and $b_{2 n+2}=-a_{2 n+2}$.

As an application of the fact that $\tau: F_{n} \rightarrow F_{n}$ is not completely contractive, we will now discuss the operator space structures induced by triple monomorphisms on Cartan factors of type 4. See the last part of Section 2 for a brief account on this class. We recall the well-known fact that for any $N \geq 1, E_{N}$ is a Cartan factor of type 4. Moreover it follows from the discussion in Section 5 that for any $n \geq 1$, the linear maps $u_{ \pm}: E_{2 n+1} \rightarrow \mathcal{C}_{2 n}$ defined by $u_{ \pm}\left(w_{2 n+1}\right)= \pm i^{n} \omega_{1} \cdots \omega_{2 n}, u_{ \pm}(1)=1$ and $u_{ \pm}\left(\omega_{j}\right)=\omega_{j}$ for $j=1, \ldots, 2 n$ are triple monomorphisms. Thus $F_{n}$ is a Cartan factor of type 4 and $\tau$ is a triple isomorphism. 
We use the opposite operator space $F_{n}^{\text {op }}$ considered in Remark 5.5 (3). We let $F_{n} \cap F_{n}^{\text {op }}$ be the 'intersection' of these two operator spaces defined by letting

$$
\|z\|_{M_{k}\left(F_{n} \cap F_{n}^{\mathrm{op}}\right)}=\max \left\{\|z\|_{M_{k}\left(F_{n}\right)},\|z\|_{M_{k}\left(F_{n}^{\mathrm{op}}\right)}\right\}, \quad k \geq 1, z \in M_{k} \otimes F_{n} .
$$

(See [18, Sect. 2.7 and 2.10].)

Proposition 7.3. Let $n \geq 1$ be an integer.

(1) Let $\mathcal{H}$ be a Hilbert space and let $u: F_{n} \rightarrow B(\mathcal{H})$ be a triple monomorphism. Then one of the following three properties holds and they mutually exclude each other. Either $u: F_{n} \rightarrow B(\mathcal{H})$ is a complete isometry; or $u: F_{n}^{\mathrm{op}} \rightarrow B(\mathcal{H})$ is a complete isometry; or $u: F_{n} \cap F_{n}^{\text {op }} \rightarrow B(\mathcal{H})$ is a complete isometry.

(2) Let $X$ be a Cartan factor of type 4, with $\operatorname{dim}(X)=2 n+2$. Then $X$ is completely isometric either to $F_{n}$ or to $E_{2 n+1}$. Furthermore, $F_{n}$ and $E_{2 n+1}$ are not completely isometric.

(3) Let $\mathcal{H}$ be a Hilbert space. Then any triple monomorphism $u: E_{2 n} \rightarrow B(\mathcal{H})$ is a complete isometry. Consequently if $X$ is a Cartan factor of type 4, with $\operatorname{dim}(X)=2 n+1$, then $X$ is completely isometric to $E_{2 n}$.

Proof. (1) Let $u: F_{n} \rightarrow B(\mathcal{H})$ be a triple monomorphism. We use the description of such mappings established in [2] and given in [3, p. 21] in terms of the socalled irreducible faithful representations. According to this description, and the relationship between $A H_{n-1}$ and $F_{n}$ discussed in Section 5, there exist Hilbert spaces $H_{1}, H_{2}$, two partial isometries $a_{1} \in B\left(H_{1}\right), a_{2} \in B(H)$, and two partial isometries

$$
U, V:\left(\Lambda_{2 n} \stackrel{2}{\otimes} H_{1}\right) \stackrel{2}{\oplus}\left(\Lambda_{2 n} \stackrel{2}{\otimes} H_{2}\right) \longrightarrow \mathcal{H}
$$

such that

$u(x)=V\left(x \otimes a_{1}, \tau(x) \otimes a_{2}\right) U^{*}$ and $V^{*} V\left(x \otimes a_{1}, \tau(x) \otimes a_{2}\right) U^{*} U=\left(x \otimes a_{1}, \tau(x) \otimes a_{2}\right)$

for any $x \in F_{n}$. This readily implies, using Remark 5.5 (3), that for any $k \geq 1$ and any $z \in M_{k} \otimes F_{n}$, we have

$$
\begin{aligned}
\left\|\left(I_{M_{k}} \otimes u\right) z\right\|_{M_{k}(B(\mathcal{H}))} & =\max \left\{\left\|a_{1}\right\|\|x\|_{M_{k}\left(F_{n}\right)},\left\|a_{2}\right\|\|\tau(x)\|_{M_{k}\left(F_{n}\right)}\right\} \\
& =\max \left\{\left\|a_{1}\right\|\|x\|_{M_{k}\left(F_{n}\right)},\left\|a_{2}\right\|\|x\|_{M_{k}\left(F_{n}^{\mathrm{op}}\right)}\right\} .
\end{aligned}
$$

Note that $\left\|a_{i}\right\| \in\{0,1\}$. If $\left\|a_{1}\right\|=1$ and $\left\|a_{2}\right\|=0$, then $u$ is a complete isometry on $F_{n}$. If $\left\|a_{1}\right\|=0$ and $\left\|a_{2}\right\|=1$, then $u$ is a complete isometry on $F_{n}^{\text {op }}$. Finally if $\left\|a_{1}\right\|=\left\|a_{2}\right\|=1$, then $u$ is a complete isometry on $F_{n} \cap F_{n}^{\text {op }}$.

The fact that these three cases mutually exclude each other simply means that the identity map is not a complete isometry from $F_{n}$ into $F_{n}^{\text {op }}$. This is the case because $\tau$ is not a complete isometry, by Proposition 7.1 .

(2) We first observe that $E_{2 n+1}$ is completely isometric to $F_{n} \cap F_{n}^{\mathrm{op}}$. This follows from our discussion in Section 5. Indeed if $\pi: \mathcal{C}_{2 n+1} \rightarrow \mathcal{C}_{2 n} \oplus{ }^{\infty} \mathcal{C}_{2 n}$ is the $*$-isomorphism given by (5.6), then $\pi\left(E_{2 n+1}\right)=\left\{(x, \tau(x)): x \in F_{n}\right\}$. Since $\pi$ is a complete isometry, the result follows at once.

Now let $X$ be a Cartan factor of type 4 , with $\operatorname{dim}(X)=2 n+2$. It follows from (1) that $X$ is completely isometric either to $F_{n}$, to $F_{n}^{\text {op }}$, or to $F_{n} \cap F_{n}^{\text {op }}$. Since $F_{n}$ 
and $F_{n}^{\text {op }}$ are completely isometric (via $\tau$ ), we deduce using the above observation that $X$ is actually completely isometric to either $F_{n}$ or $E_{2 n+1}$.

It remains to prove that $E_{2 n+1}$ is not completely isometric to $F_{n}$. We have noticed above that there is a (natural) completely isometric triple isomorphism $J: E_{2 n+1} \rightarrow F_{n} \cap F_{n}^{\text {op }}$. Let $v: E_{2 n+1} \rightarrow E_{2 n+1}$ be an arbitrary linear isometry and recall that this forces $v$ to be a triple isomorphism. Applying part (1) of this proposition to $u=v J^{-1}$, we obtain that for any $k \geq 1$ and for any $x \in M_{k} \otimes E_{2 n+1}$, $\left\|\left(I_{M_{k}} \otimes v\right) x\right\|_{M_{k}\left(E_{2 n+1}\right)}$ is equal either to $\|x\|_{M_{k}\left(E_{2 n+1}\right)}$, or to $\|J(x)\|_{M_{k}\left(F_{n}\right)}$, or to

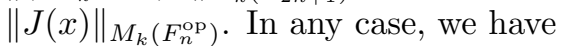

$$
\left\|\left(I_{M_{k}} \otimes u\right) x\right\|_{M_{k}\left(E_{2 n+1}\right)} \leq\|x\|_{M_{k}\left(E_{2 n+1}\right)} .
$$

Applying the same reasoning to $v^{-1}$ we obtain that $v$ is actually a complete isometry. Thus any isometry of $E_{2 n+1}$ is a complete one. Since $F_{n}$ admits an isometry which is not a complete one (namely, $\tau$ ), these two spaces cannot be completely isometric.

(3) As in (1), this follows from the description of the triple monomorphism $u: E_{2 n} \rightarrow B(\mathcal{H})$ given by [3, p. 21] (and 2]). Indeed there exist a Hilbert space $H$, a partial isometry $a \in B(H)$, and two partial isometries

$$
U, V:\left(\Lambda_{2 n} \stackrel{2}{\otimes} H\right) \longrightarrow \mathcal{H}
$$

such that

$$
u(x)=V(x \otimes a) U^{*} \quad \text { and } \quad V^{*} V(x \otimes a) U^{*} U=x \otimes a
$$

for any $x \in F_{n}$. This factorization readily implies that $u$ is a complete isometry.

Remark 7.4. Using the description of triple monomorphisms on Cartan factors of types 1-3 established in [2 and given in [3, p. 21], one obtains analogs of the above proposition for these factors, as follows.

(1) Let $H, \mathcal{H}$ be Hilbert spaces. Any triple monomorphism $\mathcal{S}(H) \rightarrow B(\mathcal{H})$ is a complete isometry. If $\operatorname{dim} H \geq 5$, any triple monomorphism $\mathcal{A}(H) \rightarrow B(\mathcal{H})$ is a complete isometry.

(2) Let $H, K$ be Hilbert spaces and assume that $\operatorname{dim}(H) \geq 2$ and $\operatorname{dim}(K) \geq 2$. Then for any triple monomorphism $u: B(H, K) \rightarrow B(\mathcal{H})$, one of the following three properties holds and they mutually exclude each other. Either $u: B(H, K) \rightarrow$ $B(\mathcal{H})$ is a complete isometry; or $u: B(H, K)^{\text {op }} \rightarrow B(\mathcal{H})$ is a complete isometry; or $u: B(H, K) \cap B(H, K)^{\text {op }} \rightarrow B(\mathcal{H})$ is a complete isometry. Furthermore if $X$ is a Cartan factor which is triple equivalent to $B(H, K)$, then $X$ is completely isometric either to $B(H, K)$ or to $B(H, K) \cap B(H, K)^{\text {op }}$. Lastly, the latter two spaces are not completely isometric.

For a partial description of all possible operator space structures induced by triple monomorphisms $B(\mathbb{C}, K) \rightarrow B(\mathcal{H})$, see [13].

We now turn to the study of $\tau: F_{n}^{p} \rightarrow F_{n}^{p}$ for finite $p$. For any integer $N \geq 1$, let $\mathbb{D}_{N}$ be the finite set $\{-1,1\}^{N}$ equipped with its uniform probability measure $\mathbb{P}$, and consider the Rademacher functions $\varepsilon_{1}, \ldots, \varepsilon_{N}: \mathbb{D}_{N} \rightarrow\{-1,1\}$ defined by 
letting $\varepsilon_{j}(\Theta)=\theta_{j}$ for any $\Theta=\left(\theta_{1}, \ldots, \theta_{N}\right)$ in $\mathbb{D}_{N}$ and any $1 \leq j \leq N$. We will need the following lemma, in which $N=2 n$ and \|\|$_{p}$ stands for the norm in $L^{p}\left(\mathbb{D}_{2 n}\right)$.

Lemma 7.5. For any complex numbers $\alpha_{0}, \alpha_{1}, \ldots, \alpha_{2 n+1}$, we have

$$
\left\|\tau: F_{n}^{p} \longrightarrow F_{n}^{p}\right\|_{c b} \geq \frac{\left\|\alpha_{0}+\sum_{j=1}^{2 n} \alpha_{j} \varepsilon_{j}+\alpha_{2 n+1} \prod_{j=1}^{2 n} \varepsilon_{j}\right\|_{p}}{\left\|\alpha_{0}+\sum_{j=1}^{2 n} \alpha_{j} \varepsilon_{j}-\alpha_{2 n+1} \prod_{j=1}^{2 n} \varepsilon_{j}\right\|_{p}} .
$$

Proof. This is a continuation of the proof of Proposition 7.1. Let $\alpha_{0}, \alpha_{1}, \ldots, \alpha_{2 n+1}$ be complex numbers. We will show that

$$
\left\|\sum_{j=0}^{2 n+1} \alpha_{j} s_{j}^{*} \otimes s_{j}\right\|_{L^{p}\left(\mathcal{C}_{2 n}\right)^{p} L^{p}\left(\mathcal{C}_{2 n}\right)}=\left\|\alpha_{0}+\sum_{j=1}^{2 n} \alpha_{j} \varepsilon_{j}+\alpha_{2 n+1} \prod_{j=1}^{2 n} \varepsilon_{j}\right\|_{p} .
$$

Changing $\alpha_{2 n+1}$ into $-\alpha_{2 n+1}$ and applying the definition of $\tau$, this implies the result.

We first note that the identification (17.2) induces an isometric isomorphism

$$
L^{p}\left(\mathcal{C}_{2 n}\right) \stackrel{p}{\otimes} L^{p}\left(\mathcal{C}_{2 n}\right) \simeq S^{p}\left(L^{2}\left(\mathcal{C}_{2 n}\right)\right),
$$

which yields

$$
\left\|\sum_{j=0}^{2 n+1} \alpha_{j} s_{j}^{*} \otimes s_{j}\right\|_{L^{p}\left(\mathcal{C}_{2 n}\right) \otimes L^{p}\left(\mathcal{C}_{2 n}\right)}=\left(\frac{1}{2^{2 n}}\right)^{\frac{1}{p}}\left\|\sum_{j=0}^{2 n+1} \alpha_{j} \pi_{j}\right\|_{S^{p}\left(L^{2}\left(\mathcal{C}_{2 n}\right)\right)} .
$$

The subspace of $S^{p}\left(L^{2}\left(\mathcal{C}_{2 n}\right)\right)$ of operators which are diagonal with respect to the orthonormal basis $\left(\omega_{A}\right)_{A \in \mathcal{P}_{2 n}}$ is equal to $\ell_{\mathcal{P}_{2 n}}^{p}$. To any $A \in \mathcal{P}_{2 n}$, let us associate the $2 n$-tuple $\Theta_{A}=\left(\theta_{1}, \ldots, \theta_{2 n}\right) \in \mathbb{D}_{2 n}$ defined by $\theta_{j}=1 \Leftrightarrow j \notin A$. Then it follows from the proof of Proposition 7.1 that

$$
\pi_{j}\left(\omega_{A}\right)=\left(\varepsilon_{j}\left(\prod_{i=1}^{2 n} \varepsilon_{i}\right)\right)\left(\Theta_{A}\right) \omega_{A}, \quad j=1, \ldots, 2 n .
$$

Thus in the isometric isomorphism $\ell_{\mathcal{P}_{2 n}}^{p} \simeq L^{p}\left(\mathbb{D}_{2 n}\right)$ induced by the correspondance $A \leftrightarrow \Theta_{A}$, the diagonal operator $\pi_{j}$ corresponds to $\varepsilon_{j}\left(\prod_{i=1}^{2 n} \varepsilon_{i}\right)$ for any $1 \leq j \leq 2 n$. Likewise, $\pi_{2 n+1}$ corresponds to $\prod_{i=1}^{2 n} \varepsilon_{i}$. Hence

$$
\left(\frac{1}{2^{2 n}}\right)^{\frac{1}{p}}\left\|\sum_{j=0}^{2 n+1} \alpha_{j} \pi_{j}\right\|_{S^{p}\left(L^{2}\left(\mathcal{C}_{2 n}\right)\right)}=\left\|\alpha_{0}+\sum_{j=1}^{2 n} \alpha_{j} \varepsilon_{j}\left(\prod_{i=1}^{2 n} \varepsilon_{i}\right)+\alpha_{2 n+1} \prod_{i=1}^{2 n} \varepsilon_{i}\right\|_{p}
$$

Now set $\eta_{j}=\varepsilon_{j}\left(\prod_{i=1}^{2 n} \varepsilon_{i}\right)$ for any $1 \leq j \leq 2 n$. Then we have

$$
\prod_{j=1}^{2 n} \eta_{j}=\prod_{j=1}^{2 n} \varepsilon_{j} \quad \text { and } \quad \varepsilon_{j}=\eta_{j}\left(\prod_{i=1}^{2 n} \eta_{i}\right), \quad 1 \leq j \leq 2 n .
$$

Consider $\left(\theta_{1}, \ldots, \theta_{2 n}\right) \in\{-1,1\}^{2 n}$ and let $\theta=\theta_{1} \theta_{2} \cdots \theta_{2 n}$ be the product of these \pm 1 . It follows from above that

$$
\begin{aligned}
\mathbb{P}\left(\left\{\eta_{j}=\theta_{j} \forall j=1, \ldots, 2 n\right\}\right) & =\mathbb{P}\left(\left\{\varepsilon_{j}=\theta_{j} \theta \forall j=1, \ldots, 2 n\right\}\right) \\
& =\mathbb{P}\left(\left\{\varepsilon_{j}=\theta_{j} \forall j=1, \ldots, 2 n\right\}\right)=\frac{1}{2^{2 n}} .
\end{aligned}
$$


Thus $\left(\eta_{1}, \ldots, \eta_{2 n}\right)$ has the same distribution as $\left(\varepsilon_{1}, \ldots, \varepsilon_{2 n}\right)$, and hence

$$
\left\|\alpha_{0}+\sum_{j=1}^{2 n} \alpha_{j} \varepsilon_{j}\left(\prod_{i=1}^{2 n} \varepsilon_{i}\right)+\alpha_{2 n+1} \prod_{i=1}^{2 n} \varepsilon_{i}\right\|_{p}=\left\|\alpha_{0}+\sum_{j=1}^{2 n} \alpha_{j} \varepsilon_{j}+\alpha_{2 n+1} \prod_{i=1}^{2 n} \varepsilon_{i}\right\|_{p} .
$$

Together with (7.5) and (7.6), this implies the equality (7.4).

Proposition 7.6. For any $n \geq 1$, we have

$$
\left\|\tau: F_{n}^{1} \longrightarrow F_{n}^{1}\right\|_{c b}=\frac{n+1}{n} .
$$

Proof. The upper estimate clearly follows from the proof of Proposition 7.1. For the lower estimate we consider

$$
f=1+\sum_{j=1}^{2 n} \varepsilon_{j}-(-1)^{n} \prod_{j=1}^{2 n} \varepsilon_{j} \quad \text { and } \quad g=1+\sum_{j=1}^{2 n} \varepsilon_{j}+(-1)^{n} \prod_{j=1}^{2 n} \varepsilon_{j}
$$

in $L^{1}\left(\mathbb{D}_{2 n}\right)$. According to Lemma 7.5 , it suffices to show that

$$
\frac{\|f\|_{1}}{\|g\|_{1}}=\frac{n+1}{n}
$$

For any $i=1, \ldots, 2 n$, let $\rho_{i}: L^{1}\left(\mathbb{D}_{2 n}\right) \rightarrow L^{1}\left(\mathbb{D}_{2 n}\right)$ be induced by the $*$-representation which takes $\varepsilon_{i}$ to $-\varepsilon_{i}$ and which takes $\varepsilon_{j}$ to $\varepsilon_{j}$ for any $j \neq i$. Then $\rho_{i}$ is an isometry and

$$
\rho_{i}\left(\prod_{j=1}^{2 n} \varepsilon_{j}\right)=-\prod_{j=1}^{2 n} \varepsilon_{j}
$$

Then we let $\rho=-\rho_{1} \circ \cdots \circ \rho_{2 n}$. A few elementary computations (left to the reader) yield

$$
2 n g=f+\sum_{i=1}^{2 n} \rho_{i}(f)+\rho(f) .
$$

Let $k$ be the $\mathbb{Z}$-valued function on $\mathbb{D}_{2 n}$ defined as

$$
k=\operatorname{Card}\left\{j \in\{1, \ldots, 2 n\}: \varepsilon_{j}=1\right\}
$$

and let $m=k-n$. Then

$$
f=1+k-(2 n-k)-(-1)^{n}(-1)^{2 n-k}=1-(-1)^{m}+2 m ;
$$

hence $f$ is valued in $4 \mathbb{Z}$. Moreover for any $i=1, \ldots, 2 n$, we have

$$
f-\rho_{i}(f)=2\left(\varepsilon_{i}-\prod_{j=1}^{2 n} \varepsilon_{j}\right)
$$

hence $f-\rho_{i}(f)$ is valued in $\{-4,0,4\}$. Consequently, $f$ and $\rho_{i}(f)$ have the same sign everywhere on $\mathbb{D}_{2 n}$. Likewise $f$ and $\rho(f)$ have the same sign. This implies that

$$
\left\|f+\sum_{i=1}^{2 n} \rho_{i}(f)+\rho(f)\right\|_{1}=\|f\|_{1}+\sum_{i=1}^{2 n}\left\|\rho_{i}(f)\right\|_{1}+\|\rho(f)\|_{1} .
$$

We deduce that $2 n\|g\|_{1}=(2 n+2)\|f\|_{1}$, which concludes the proof. 
Theorem 7.7. Let $n \geq 1$ be an integer and let $1 \leq p<\infty$. The following are equivalent.

(i) $\left\|\tau: F_{n}^{p} \rightarrow F_{n}^{p}\right\|_{c b} \leq 1$ (equivalently, $\tau: F_{n}^{p} \rightarrow F_{n}^{p}$ is a complete isometry).

(ii) $p$ is an even integer and $2 n \geq p$.

Proof. (ii) $\Rightarrow$ (i): Assume that $p=2 q, q$ is an integer and $q \leq n$. Again we use the notation (7.1). To prove (i), we consider $a_{0}, a_{1}, \ldots, a_{2 n+1}$ in $S^{p}$ and aim at showing that

$$
\left\|\sum_{j=0}^{2 n} a_{j} \otimes s_{j}+a_{2 n+1} \otimes s_{2 n+1}\right\|_{S^{p}\left[L^{p}\left(\mathcal{C}_{2 n}\right)\right]}^{p}=\left\|\sum_{j=0}^{2 n} a_{j} \otimes s_{j}-a_{2 n+1} \otimes s_{2 n+1}\right\|_{S^{p}\left[L^{p}\left(\mathcal{C}_{2 n}\right)\right]}^{p} .
$$

We have

$$
\begin{aligned}
\left|\sum_{j=0}^{2 n+1} a_{j} \otimes s_{j}\right|^{2 q} & =\left(\sum_{j=0}^{2 n+1} a_{j} \otimes s_{j}\right)^{*}\left(\sum_{j=0}^{2 n+1} a_{j} \otimes s_{j}\right) \cdots\left(\sum_{j=0}^{2 n+1} a_{j} \otimes s_{j}\right)^{*}\left(\sum_{j=0}^{2 n+1} a_{j} \otimes s_{j}\right) \\
& =\sum_{0 \leq j_{1}, \ldots, j_{2 q} \leq 2 n+1} a_{j_{1}}^{*} a_{j_{2}} \ldots a_{j_{2 q-1}}^{*} a_{j_{2 q}} \otimes s_{j_{1}}^{*} s_{j_{2}} \ldots s_{j_{2 q-1}}^{*} s_{j_{2 q}} .
\end{aligned}
$$

Here the sum runs over all $\left(j_{1}, \ldots, j_{2 q}\right) \in\{0, \ldots, 2 n+1\}^{2 q}$. Recall that $T r$ and $t r$ denote the canonical traces on $\mathcal{C}_{2 n}$ and $B\left(\ell^{2}\right)$, respectively. By the above calculation, the left hand side of (7.7) is equal to

$$
\sum_{0 \leq j_{1}, \ldots, j_{2 q} \leq 2 n+1} \operatorname{tr}\left(a_{j_{1}}^{*} a_{j_{2}} \ldots a_{j_{2 q-1}}^{*} a_{j_{2 q}}\right) \operatorname{Tr}\left(s_{j_{1}}^{*} s_{j_{2}} \ldots s_{j_{2 q-1}}^{*} s_{j_{2 q}}\right) .
$$

Changing $a_{2 n+1}$ into $-a_{2 n+1}$, we see that the right hand side of (17.7) is equal to

$$
\sum_{0 \leq j_{1}, \ldots, j_{2 q} \leq 2 n+1}(-1)^{\left|\left\{k: j_{k}=2 n+1\right\}\right|} \operatorname{tr}\left(a_{j_{1}}^{*} a_{j_{2}} \ldots a_{j_{2 q-1}}^{*} a_{j_{2 q}}\right) \operatorname{Tr}\left(s_{j_{1}}^{*} s_{j_{2}} \ldots s_{j_{2 q-1}}^{*} s_{j_{2 q}}\right) .
$$

To show the equality (7.7) it therefore suffices to check that if a $2 q$-tuple $\left(j_{1}, \ldots, j_{2 q}\right)$ in $\{0, \ldots, 2 n+1\}^{2 q}$ is such that the cardinality $\left|\left\{k: j_{k}=2 n+1\right\}\right|$ is an odd number, then

$$
\operatorname{Tr}\left(s_{j_{1}}^{*} s_{j_{2}} \ldots s_{j_{2 q-1}}^{*} s_{j_{2 q}}\right)=0 .
$$

Suppose that $\left|\left\{k: j_{k}=2 n+1\right\}\right|=2 m+1$, for some integer $m \geq 0$. Recall that $s_{j}^{*}=s_{j}$ for any $j \leq 2 n$, that $s_{2 n+1}^{*}=(-1)^{n} s_{2 n+1}$ and that for any $0 \leq j, j^{\prime} \leq$ $2 n+1$, the operators $s_{j}$ and $s_{j^{\prime}}$ either commute or anticommute. Note also that $s_{2 n+1}^{2}=(-1)^{n}$. Thus we have

$$
\begin{aligned}
\left|\operatorname{Tr}\left(s_{j_{1}}^{*} s_{j_{2}} \ldots s_{j_{2 q-1}}^{*} s_{j_{2 q}}\right)\right| & =\left|\operatorname{Tr}\left(s_{j_{1}} s_{j_{2}} \ldots s_{j_{2 q-1}} s_{j_{2 q}}\right)\right| \\
& =\left|\operatorname{Tr}\left(s_{2 n+1}^{2 m+1} S\right)\right|=\left|\operatorname{Tr}\left(s_{2 n+1} S\right)\right|,
\end{aligned}
$$

where $S \in \mathcal{C}_{2 n}$ is the product of $(2 q-(2 m+1))$ operators belonging to the set $\left\{1, \omega_{1}, \ldots, \omega_{2 n}\right\}$. Since $q \leq n$, this product has at most $(2 n-1)$ factors. Since $\omega_{j}^{2}=1$ for any $j \leq 2 n$, we deduce that there exists an integer $r \geq 1$ and $r$ distinct integers $i_{1}, \ldots, i_{r}$ between 1 and $2 n$ such that $s_{2 n+1} S=\omega_{i_{1}} \cdots \omega_{i_{r}}$. Then the trace of $s_{2 n+1} S$ is zero. 
(i) $\Rightarrow$ (ii): Let us assume that $p=2 q, q$ is an integer, $q>n$, and let us show that $\tau: F_{n}^{p} \rightarrow F_{n}^{p}$ is not completely contractive. We set

$$
P(t)=\left\|1+\sum_{j=1}^{2 n} \varepsilon_{j}+t \prod_{j=1}^{2 n} \varepsilon_{j}\right\|_{p}^{p}, \quad t \in \mathbb{R} .
$$

Then $P$ is a polynomial and according to Lemma 7.5, the fact that $\tau: F_{n}^{p} \rightarrow F_{n}^{p}$ is not completely contractive is equivalent to $P$ not being even. Let $c_{1}=P^{\prime}(0)$ be the coefficient of degree 1 . Set $\varepsilon_{0}=1$ for convenience. Then by a computation similar to the one in the first part of this proof, one obtains that

$$
c_{1}=\sum_{\left(j_{1}, \ldots, j_{2 q}\right) \in \Gamma} \mathbb{E}\left(\prod_{j=1}^{2 n} \varepsilon_{j} \prod_{k: j_{k} \neq 2 n+1} \varepsilon_{j_{k}}\right),
$$

where $\Gamma \subset\{0, \ldots, 2 n+1\}^{2 q}$ is the set of all $2 q$-tuples $\left(j_{1}, \ldots, j_{2 q}\right)$ for which there is a unique $1 \leq k \leq 2 q$ such that $j_{k}=2 n+1$. Here $\mathbb{E}$ denotes the conditional expectation on $\left(\mathbb{D}_{2 n}, \mathbb{P}\right)$. Observe that for any $\left(j_{1}, \ldots, j_{2 q}\right) \in \Gamma$,

$$
\mathbb{E}\left(\prod_{j=1}^{2 n} \varepsilon_{j} \prod_{k: j_{k} \neq 2 n+1} \varepsilon_{j_{k}}\right)=0 \text { or } 1 \text {. }
$$

Moreover for the $2 q$-tuple defined by letting $j_{k}=k$ for any $1 \leq k \leq 2 n+1$ and $j_{k}=0$ for any $k \geq 2 n+2$, then the above conditional expectation is equal to 1 . (We use that $q>n$ to define this particular $2 q$-tuple.) We deduce that $c_{1}>0$, and hence that $P$ is not even.

Let us now assume that $\gamma=\frac{p}{2}$ is not an integer and let us show that $\tau$ is not a complete contraction on $F_{n}^{p}$. For any positive real number $a>0$ and any $t \in \mathbb{R}$, we set

$$
\Phi(a, t)=\left\|1+i a^{\frac{1}{2}}\left(1+\sum_{j=1}^{2 n} \varepsilon_{j}+t \prod_{j=1}^{2 n} \varepsilon_{j}\right)\right\|_{p}^{p} .
$$

Using Lemma 7.5 again, it suffices to show that for some positive real number $a>0$, the function $\Phi(a, \cdot)$ is not even. We have

$$
\Phi(a, t)=\mathbb{E}\left[\left(1+a\left(1+\sum_{j=1}^{2 n} \varepsilon_{j}+t \prod_{j=1}^{2 n} \varepsilon_{j}\right)^{2}\right)^{\gamma}\right]
$$

hence $\Phi$ extends to a $C^{\infty}$ function on a neighborhood of zero.

Suppose that $\Phi(a, \cdot)$ is even for any $a>0$. Then $\frac{\partial^{n+1} \Phi}{\partial a^{n+1}}(0, \cdot)$ also is an even function. On a neighborhood of zero, we have

$$
\begin{aligned}
& \frac{\partial^{n+1} \Phi}{\partial a^{n+1}}(a, t) \\
& =\delta_{\gamma, n} \mathbb{E}\left[\left(1+\sum_{j=1}^{2 n} \varepsilon_{j}+t \prod_{j=1}^{2 n} \varepsilon_{j}\right)^{2(n+1)}\left(1+a\left(1+\sum_{j=1}^{2 n} \varepsilon_{j}+t \prod_{j=1}^{2 n} \varepsilon_{j}\right)^{2}\right)^{\gamma-(n+1)}\right],
\end{aligned}
$$

where $\delta_{\gamma, n}=\gamma(\gamma-1) \cdots(\gamma-n)$. Since $\gamma$ is not an integer, this constant is nonzero and we obtain that for any $t \in \mathbb{R}$,

$$
\delta_{\gamma, n}^{-1} \frac{\partial^{n+1} \Phi}{\partial a^{n+1}}(0, t)=\mathbb{E}\left[\left(1+\sum_{j=1}^{2 n} \varepsilon_{j}+t \prod_{j=1}^{2 n} \varepsilon_{j}\right)^{2(n+1)}\right]
$$


When we showed above that $\tau: F_{n}^{2(n+1)} \rightarrow F_{n}^{2(n+1)}$ is not a complete contraction, we showed that the above function of $t$ is not even. Hence we obtain a contradiction.

We now give an application to an extension problem.

Corollary 7.8. Let $p \geq 4$ be an even integer. There exist a subspace $X \subset S^{p}$ and a completely bounded map $u: X \rightarrow S^{p}$ which has no bounded extension $S^{p} \rightarrow S^{p}$.

We will need the following classical averaging argument. Let $Z$ be a reflexive Banach space, let $G$ be an amenable group and let $\lambda: G \rightarrow B(Z)$ be a strongly continuous representation such that $\lambda(g): Z \rightarrow Z$ is a (necessarily onto) isometry for any $g \in G$. We say that a subspace $X \subset Z$ is invariant if $\lambda(g)$ maps $X$ into $X$ for any $g \in G$ and we say that a bounded linear map $u: X \rightarrow Z$ is a multiplier if

$$
\lambda(g)(u(x))=u(\lambda(g) x), \quad x \in X
$$

Lemma 7.9. Let $v: Z \rightarrow Z$ be a bounded linear map, and assume that $X \subset Z$ is invariant and that $v_{\mid X}: X \rightarrow Z$ is a multiplier. Then there exist a multiplier $w: Z \rightarrow Z$ such that $\|w\| \leq\|v\|$ and $v_{\mid X}=w_{\mid X}$.

Proof. Let $\psi \in L^{\infty}(G)^{*}$ be a translation invariant mean on $G$. For any $x \in Z$ and $y \in Z^{*}$, consider the function

$$
f_{x, y}(g)=\left\langle\lambda\left(g^{-1}\right)(v(\lambda(g) x)), y\right\rangle, \quad g \in G .
$$

Then we may define $w \in B(Z)$ by letting

$$
\langle w(x), y\rangle=\psi\left(f_{x, y}\right)
$$

and it is clear that the operator $w$ satisfies the required conditions.

Proof of Corollary 7.8. Let $p \geq 4$ be an even integer and let $n=p / 2$. We let $p^{\prime}$ be the conjugate number of $p$ and for $q \in\left\{p, p^{\prime}\right\}$, we let $\tau_{q}: F_{n}^{q} \rightarrow F_{n}^{q}$ denote the transposition. According to Theorem 7.7, we have $\left\|\tau_{p}\right\|_{c b}=1$ whereas $c=\left\|\tau_{p^{\prime}}\right\|_{c b}>$ 1.

We fix an integer $k \geq 1$ and we consider

$$
Z_{k, q}=L^{q}\left(\mathcal{C}_{2 n}\right) \stackrel{q}{\otimes} \cdots \stackrel{q}{\otimes} L^{q}\left(\mathcal{C}_{2 n}\right) \stackrel{q}{\otimes} S^{q} \quad \text { and } \quad X_{k, q}=F_{n}^{q} \stackrel{q}{\otimes} \cdots \stackrel{q}{\otimes} F_{n}^{q} \stackrel{q}{\otimes} S^{q} .
$$

We will exhibit a complete contraction $u: X_{k, p} \rightarrow Z_{k, p}$ such that $\|v\| \geq c^{k}$ for any bounded linear map $v: Z_{k, p} \rightarrow Z_{k, p}$ extending $u$. Since $Z_{k, p}$ is completely isometric to $S^{p}$, the result follows at once using a standard direct sum argument.

For any $\Theta=\left(\theta_{1}, \ldots, \theta_{2 n}\right) \in \mathbb{D}_{2 n}$, we let $\pi_{\Theta}: L^{q}\left(\mathcal{C}_{2 n}\right) \rightarrow L^{q}\left(\mathcal{C}_{2 n}\right)$ denote the $L^{q}$ version of the $*$-representation $\mathcal{C}_{2 n} \rightarrow \mathcal{C}_{2 n}$ taking $\omega_{j}$ to $\theta_{j} \omega_{j}$ for any $j=1, \ldots, 2 n$. This is an isometry and $\Theta \mapsto \pi_{\Theta}$ is a representation of $\mathbb{D}_{2 n}$ on $L^{q}\left(\mathcal{C}_{2 n}\right)$. Then let $\mathbb{T}$ be the unit circle and consider elements of $S^{q}$ as infinite matrices $\left[t_{r s}\right]_{r, s \geq 1}$ in the usual way. For any $\alpha=\left(\alpha_{r}\right)_{r \geq 1}$ and $\beta=\left(\beta_{s}\right)_{s \geq 1}$ in $\mathbb{T}^{\infty}$, let $\gamma(\alpha, \beta): S^{q} \rightarrow S^{q}$ be the linear mapping taking any matrix $\left[t_{r s}\right] \in S^{q}$ to $\left[\alpha_{r} t_{r s} \beta_{s}\right]$. Then $\gamma: \mathbb{T}^{\infty} \times \mathbb{T}^{\infty} \rightarrow$ $B\left(S^{q}\right)$ is a strongly continuous isometric representation. We will apply Lemma 7.9 to the group

$$
G=\underbrace{\mathbb{D}_{2 n} \times \cdots \times \mathbb{D}_{2 n}}_{k \text { times }} \times \mathbb{T}^{\infty} \times \mathbb{T}^{\infty}
$$

and to the representation $\lambda: G \rightarrow B\left(Z_{k, q}\right)$ defined by letting

$$
\lambda\left(\Theta^{1}, \ldots, \Theta^{k}, \alpha, \beta\right)=\pi_{\Theta^{1}} \otimes \cdots \otimes \pi_{\Theta^{k}} \otimes \gamma(\alpha, \beta) .
$$


Indeed, $G$ is amenable and it follows from the above discussion that $\lambda$ is a strongly continuous isometric representation. Let $E_{r s}$ denote the canonical matrix units in $S^{q}$. It is easy to check (left to the reader) that a bounded linear map $w: Z_{k, q} \rightarrow Z_{k, q}$ is a multiplier if and only if $w$ is diagonal with respect to the elements $\omega_{A_{1}} \otimes \cdots \otimes$ $\omega_{A_{k}} \otimes E_{r s}$, for $A_{1}, \ldots A_{k} \in \mathcal{P}_{2 n}$ and $r, s \geq 1$.

Clearly the space $X_{k, p}$ is invariant. Let

$$
u=\tau_{p} \otimes \cdots \otimes \tau_{p} \otimes I_{S^{p}}: X_{k, p} \longrightarrow Z_{k, p} .
$$

Then $u$ is a multiplier and $\|u\|_{c b} \leq 1$. Let $v: Z_{k, q} \rightarrow Z_{k, q}$ be a bounded extension of $u$. By Lemma 7.9 there exist a multiplier $w: Z_{k, q} \rightarrow Z_{k, q}$ extending $u$ and such that $\|w\| \leq\|v\|$. Now consider the adjoint map $w^{*}: Z_{k, p^{\prime}} \rightarrow Z_{k, p^{\prime}}$. Let

$$
\mathcal{F}=\{\emptyset,\{1\}, \ldots,\{2 n\},\{1, \ldots, 2 n\}\}
$$

and recall that $F_{n}$ is the linear span of $\left\{\omega_{A}: A \in \mathcal{F}\right\}$. For any $A_{1}, \ldots, A_{k} \in \mathcal{F}$ and any $r, s \geq 1$, we have

$$
\begin{aligned}
\left\langlew ^ { * } \left(\omega_{A_{1}} \otimes \cdots\right.\right. & \left.\left.\otimes \omega_{A_{k}} \otimes E_{r s}\right), \omega_{A_{1}} \otimes \cdots \otimes \omega_{A_{k}} \otimes E_{r s}\right\rangle \\
& =\left\langle\omega_{A_{1}} \otimes \cdots \otimes \omega_{A_{k}} \otimes E_{r s}, \tau\left(\omega_{A_{1}}\right) \otimes \cdots \otimes \tau\left(\omega_{A_{k}}\right) \otimes E_{r s}\right\rangle \\
& =(-1)^{m},
\end{aligned}
$$

where $m$ is the number of $j$ 's such that $A_{j}=\{1, \ldots, 2 n\}$. Since $w$ is a multiplier, $w^{*}$ is a multiplier as well; hence we deduce from above that

$$
w^{*}\left(\omega_{A_{1}} \otimes \cdots \otimes \omega_{A_{k}} \otimes E_{r s}\right)=(-1)^{m} \omega_{A_{1}} \otimes \cdots \otimes \omega_{A_{k}} \otimes E_{r s} .
$$

This shows that

$$
w_{\mid X_{k, p^{\prime}}}^{*}=\tau_{p^{\prime}} \otimes \cdots \otimes \tau_{p^{\prime}} \otimes I_{S^{p^{\prime}}}
$$

Consequently we have

$$
c^{k}=\left\|\tau_{p^{\prime}} \otimes \cdots \otimes \tau_{p^{\prime}}\right\|_{c b}=\left\|\tau_{p^{\prime}} \otimes \cdots \otimes \tau_{p^{\prime}} \otimes I_{S^{p^{\prime}}}\right\| \leq\left\|w^{*}\right\|=\|w\| \leq\|v\|,
$$

and this concludes the proof.

We mention that instead of Theorem 7.7 one can use some results from 15 to prove the above corollary.

As a complement we will prove that Corollary 7.8 extends to the case $p=1$. We start with a simple consequence of the noncommutative Khintchine inequalities. We refer e.g. to [18, Sect. 9.8] for these inequalities. In the sequel we let

$$
\Phi_{N}=\operatorname{Span}\left\{\omega_{1}, \ldots, \omega_{N}\right\} \subset \mathcal{C}_{N}
$$

and we let $\Phi_{N}^{1}$ be that space regarded as a subspace of $L^{1}\left(\mathcal{C}_{N}\right)$. Also we let $\operatorname{Rad}_{q}^{N}=\operatorname{Span}\left\{\varepsilon_{1}, \ldots, \varepsilon_{N}\right\} \subset L^{q}\left(\mathbb{D}_{N}\right)$ for $q \in\{1, \infty\}$.

Lemma 7.10. There is a constant $C \geq 1$ such that for any $N \geq 1$ and for any $a_{1}, \ldots, a_{N}$ in $S^{1}$, we have

$$
C^{-1}\left\|\sum_{j=1}^{N} a_{j} \otimes \omega_{j}\right\|_{S^{1} \otimes \Phi_{N}^{1}} \leq\left\|\sum_{j=1}^{N} a_{j} \otimes \varepsilon_{j}\right\|_{S^{1} \otimes \operatorname{Rad}_{N}^{1}} \leq C\left\|\sum_{j=1}^{N} a_{j} \otimes \omega_{j}\right\|_{S^{1} \otimes \Phi_{N}^{1}} .
$$

Proof. This result is a simple consequence of [8, Thm. 3.7], which is more general. We give a specific proof of independent interest. We will use classical notation and techniques from operator space theory (see 18 ). The symbol $\approx$ will stand 
for a complete isomorphism whose isomorphism constants do not depend on the dimension. With this notation, the result to be proved is that

$$
\Phi_{N}^{1} \approx \operatorname{Rad}_{1}^{N} .
$$

The noncommutative Khintchine inequalities on $S^{1}$ mean that $\left(\operatorname{Rad}_{1}^{N}\right)^{*} \approx R_{N} \cap$ $C_{N}$. This implies that

$$
\left(L^{1}\left(\mathcal{C}_{N}\right) \stackrel{1}{\otimes} \operatorname{Rad}_{N}^{1}\right)^{*} \approx \mathcal{C}_{N} \otimes_{\min }\left(R_{N} \cap C_{N}\right) .
$$

For $q \in\{1, \infty\}$, we let

$$
\lambda: \mathbb{D}_{N} \longrightarrow B\left(L^{q}\left(\mathbb{D}_{N}\right)\right) \quad \text { and } \quad \mu: \mathbb{D}_{N} \longrightarrow B\left(L^{q}\left(\mathcal{C}_{N}\right)\right)
$$

be the natural representations of $\mathbb{D}_{N}$. Namely for any $\Theta=\left(\theta_{1}, \ldots, \theta_{N}\right) \in \mathbb{D}_{N}$, $\lambda(\Theta)$ (resp. $\mu(\Theta)$ ) is the $*$-representation taking $\varepsilon_{j}$ to $\theta_{j} \varepsilon_{j}$ (resp. $\omega_{j}$ to $\theta_{j} \omega_{j}$ ) for any $j$. These maps are complete isometries.

Let $\left(e_{1}, \ldots, e_{N}\right)$ denote the canonical basis of $R_{N} \cap C_{N}$, let $Z_{N}^{1}$ be the linear span of the $\omega_{j} \otimes \varepsilon_{j}$ in $L^{1}\left(\mathcal{C}_{N}\right) \stackrel{1}{\otimes} \operatorname{Rad}_{N}^{1}$ and let $Z_{N}^{\infty}$ be the linear span of the $\omega_{j} \otimes e_{j}$ in $\mathcal{C}_{N} \otimes_{\min }\left(R_{N} \cap C_{N}\right)$. Let $P: L^{1}\left(\mathcal{C}_{N}\right) \stackrel{1}{\otimes} \operatorname{Rad}_{N}^{1} \rightarrow L^{1}\left(\mathcal{C}_{N}\right) \stackrel{1}{\otimes} \operatorname{Rad}_{N}^{1}$ be the orthogonal projection onto $Z_{N}^{1}$. It is easy to check that

$$
P=\int_{\mathbb{D}_{N}} \mu(\Theta) \otimes \lambda(\Theta) d \mathbb{P}(\Theta),
$$

where $\mathbb{P}$ is the uniform probability on $\mathbb{D}_{N}$. Hence $P$ is completely contractive. Passing to the adjoints and using (7.8), this implies

$$
Z_{N}^{1 *} \approx Z_{N}^{\infty} \text {. }
$$

For any $a_{1}, \ldots, a_{N} \in B(H)$, we have

$$
\sum_{j=1}^{N}\left(a_{j} \otimes \omega_{j}\right)^{*}\left(a_{j} \otimes \omega_{j}\right)=\sum_{j=1}^{N} a_{j}^{*} a_{j} \otimes \omega_{j}^{*} \omega_{j}=\left(\sum_{j=1}^{N} a_{j}^{*} a_{j}\right) \otimes 1,
$$

and similarly, $\sum_{j}\left(a_{j} \otimes \omega_{j}\right)\left(a_{j} \otimes \omega_{j}\right)^{*}$ is equal to $\left(\sum_{j} a_{j} a_{j}^{*}\right) \otimes 1$. This implies that

$$
\left\|\sum_{j=1}^{N} a_{j} \otimes \omega_{j} \otimes e_{j}\right\|_{B(H) \otimes_{\min } \mathcal{C}_{N} \otimes_{\min }\left(R_{N} \cap C_{N}\right)}=\left\|\sum_{j=1}^{N} a_{j} \otimes e_{j}\right\|_{B(H) \otimes_{\min }\left(R_{N} \cap C_{N}\right)} .
$$

Thus $Z_{N}^{\infty}$ is completely isometrically isomorphic to $R_{N} \cap C_{N}$.

Now let $a_{1}, \ldots, a_{N} \in S^{1}$. We have $\left\|\sum_{j} a_{j} \otimes \omega_{j}\right\|_{S^{1} \otimes \Phi_{N}^{1}}=\left\|\sum_{j} a_{j} \otimes \theta_{j} \omega_{j}\right\|_{S^{1} \otimes \Phi_{N}^{1}}$ for any $\Theta \in \mathbb{D}_{N}$. Taking the average, this implies that

$$
\left\|\sum_{j=1}^{N} a_{j} \otimes \omega_{j}\right\|_{S^{1} \stackrel{1}{\otimes} \Phi_{N}^{1}}=\left\|\sum_{j=1}^{N} a_{j} \otimes \omega_{j} \otimes \varepsilon_{j}\right\|_{S^{1} \stackrel{1}{\otimes} \Phi_{N}^{1} \stackrel{1}{\otimes} \operatorname{Rad}_{N}^{1}} .
$$

Thus $Z_{N}^{1}$ is completely isometrically isomorphic to $\Phi_{N}^{1}$. Consequently, $\Phi_{N}^{1 *} \approx R_{N} \cap$ $C_{N}$, and hence $\Phi_{N}^{1} \approx \operatorname{Rad}_{N}^{1}$.

Proposition 7.11. There exist a subspace $X \subset \ell^{1}$ and a completely bounded map $u: X \rightarrow S^{1}$ which has no bounded extension $\ell^{1} \rightarrow S^{1}$. 
Proof. Suppose that this statement is false and for any $N \geq 1$, let $u_{N}: \operatorname{Rad}_{1}^{N} \rightarrow$ $L^{1}\left(\mathcal{C}_{N}\right)$ be the linear mapping taking $\varepsilon_{j}$ to $\omega_{j}$ for any $j=1, \ldots, N$. Then there is a constant $K \geq 1$ (not depending on $N$ ) such that $u_{N}$ has an extension $v_{N}: L^{1}\left(\mathbb{D}_{N}\right) \rightarrow$ $L^{1}\left(\mathcal{C}_{N}\right)$ satisfying $\left\|v_{N}\right\| \leq K\left\|u_{N}\right\|_{c b}$. The argument in Lemma 7.9 shows that $u_{N}$ actually has an extension $w_{N}: L^{1}\left(\mathbb{D}_{N}\right) \rightarrow L^{1}\left(\mathcal{C}_{N}\right)$ such that $w_{N} \circ \lambda(\Theta)=\mu(\Theta) \circ w_{N}$ for any $\Theta \in \mathbb{D}_{N}$, and $\left\|w_{N}\right\| \leq\left\|v_{N}\right\|$. Arguing as in Corollary 7.8 we find that the restriction of $w_{N}^{*}$ to $\Phi_{N}$ coincides with the canonical mapping $\Phi_{N} \rightarrow \operatorname{Rad}_{N}^{\infty}$ which takes $\omega_{j}$ to $\varepsilon_{j}$ for any $j$. Since $\Phi_{N}=\ell_{N}^{2}$ and $\operatorname{Rad}_{N}^{\infty}=\ell_{N}^{1}$ isometrically we find that

$$
\sqrt{N}=\left\|I d: \ell_{N}^{2} \longrightarrow \ell_{N}^{1}\right\| \leq\left\|w_{N}\right\| \leq K\left\|u_{N}\right\|_{c b} .
$$

However Lemma 7.10 ensures that $\sup _{N}\left\|u_{N}\right\|_{c b}<\infty$, which yields a contradiction.

We proved in Proposition 5.6 that the orthogonal projection onto $E_{N}$ is not completely contractive. For completeness we shall now give an asymptotic estimate of its completely bounded norm, based on Lemma 7.10, For any two sequences $\left(\alpha_{N}\right)_{N \geq 1}$ and $\left(\beta_{N}\right)_{N \geq 1}$ of positive real numbers, we write $\alpha_{N} \asymp \beta_{N}$ to say that there exists a constant $C \geq 1$ such that $C^{-1} \alpha_{N} \leq \beta_{N} \leq C \alpha_{N}$ for any $N \geq 1$.

Corollary 7.12. Let $P_{N}: \mathcal{C}_{N} \rightarrow \mathcal{C}_{N}$ be the orthogonal projection onto $E_{N}$. Then

$$
\left\|P_{N}\right\|_{c b} \asymp \sqrt{N} \text {. }
$$

Proof. Let $W_{N}: \mathcal{C}_{N} \rightarrow \Phi_{N} \hookrightarrow \mathcal{C}_{N}$ be the orthogonal projection onto $\Phi_{N}$. It is clear that $\left\|P_{N}\right\|_{c b} \asymp\left\|W_{N}\right\|_{c b}$. The adjoint $W_{N}^{*}: \Phi_{N}^{*} \rightarrow L^{1}\left(\mathcal{C}_{N}\right)$ coincides with the 'identity mapping' from $\Phi_{N}^{*}$ onto $\Phi_{N}^{1}$. By Lemma 7.10 and the noncommutative Khintchine inequalities, we have $\Phi_{N}^{1} \approx \operatorname{Rad}_{N}^{1} \approx\left(R_{N} \cap C_{N}\right)^{*}$; hence $\left\|W_{N}^{*}\right\|_{c b} \asymp$ $\left\|I d: \Phi_{N}^{*} \rightarrow\left(R_{N} \cap C_{N}\right)^{*}\right\|_{c b}$. Thus by duality,

$$
\left\|W_{N}\right\|_{c b} \asymp\left\|I d: R_{N} \cap C_{N} \longrightarrow \Phi_{N}\right\|_{c b} .
$$

It is shown in [18, p. 221] that $\left\|I d: R_{N} \cap C_{N} \rightarrow \Phi_{N}\right\|_{c b} \asymp \sqrt{N}$, and this estimate completes the proof.

By an averaging argument it is easy to see that for any projection $Q: \mathcal{C}_{N} \rightarrow \mathcal{C}_{N}$ whose range is equal to $E_{N}$, we have $\left\|P_{N}\right\|_{c b} \leq\|Q\|_{c b}$. Thus the 'completely bounded projection constant' of $E_{N}$ is $\asymp \sqrt{N}$. This result appeared in [8, Cor. $3.12]$.

Remark 7.13. We do not know which numbers $p \in[1,2) \cup(2, \infty)$ have the property $(\mathcal{E})$ that there exist a subspace $X \subset S^{p}$ and a completely bounded map $X \rightarrow S^{p}$ without any bounded extension $S^{p} \rightarrow S^{p}$. We just proved that $(\mathcal{E})$ holds true for any $p$ belonging to the set $B=\{1\} \cup\{2 k: k \geq 2\}$.

Let $d_{c b}$ denote the completely bounded Banach-Mazur distance of operator spaces (see [18, p. 20]). For any $m \geq 1$, the function $(p, q) \mapsto d_{c b}\left(S_{m}^{p}, S_{m}^{q}\right)$ is continuous. A thorough look at the proofs of Proposition 7.11 and Corollary 7.8 together with a simple continuity argument based on the above fact therefore shows that any $p \in B$ admits a neighborhood $\mathcal{V}_{p}$ such that $(\mathcal{E})$ holds true for any $q \in \mathcal{V}_{p}$.

\section{ACKNOWLEDGEMENTS}

We thank the referee for bringing the paper 8 to our attention. 


\section{REFERENCES}

[1] J. Arazy and Y. Friedman, The isometries of $C_{p}^{n, m}$ into $C_{p}$, Israël J. Math. 26 (1977), no. 2, 151-165. MR0440396 (55:13271)

[2] J. Arazy and Y. Friedman, Contractive projections in $C_{1}$ and $C_{\infty}$, Mem. Amer. Math. Soc. 200 (1978), 1-165. MR 481219 (82b:47023)

[3] J. Arazy and Y. Friedman, Contractive projections in $C_{p}$, Mem. Amer. Math. Soc. 459 (1992), 1-109 MR 1086564(92f:47033)

[4] O. Bratelli and D. Robinson, Operator algebras and quantum statistical mechanics II, Springer-Verlag, 1981. MR611508 (82k:82013)

[5] E. Effros and Z.-J. Ruan, Operator spaces, London Math. Soc. Monographs, Oxford University Press, 2000. MR 1793753 (2002a:46082)

[6] E. Effros and Z.-J. Ruan, Representation of operator bimodules and their applications, J. Operator Theory 19 (1988), 137-157. MR950830(91e:46077)

[7] L. Harris, Bounded symmetric homogeneous domains in infinite dimensional spaces, pp. 1340 in "Infinite dimensional holomorphy", Lecture Notes in Math. 364, Springer-Verlag, 1974. MR 0407330 (53:11106)

[8] M. Junge and T. Oikhberg, Unconditional basic sequences and homogeneous Hilbertian subspaces of non-commutative $L_{p}$ spaces, Indiana Univ. Math. J. 56 (2007), 733-765. MR2317544

[9] M. Junge, Z.-J. Ruan, and D. Sherman, A classification for 2-isometries of noncommutative $L_{p}$-spaces, Israël J. Math. 150 (2005), 285-314. MR2255812

[10] C. Le Merdy and B. Magajna, A factorization problem for normal completely bounded mappings, J. Funct. Anal. 181 (2001), 313-345. MR1821700 (2001m:46127)

[11] M. Neal and B. Russo, Contractive projections and operator spaces, Trans. Amer. Math. Soc. 355 (2003), 2223-2362. MR.1973989 (2004c:46117)

[12] M. Neal and B. Russo, Representation of contractively complemented Hilbertian operator spaces on the Fock space, Proc. Amer. Math. Soc. 134 (2006), 475-485. MR2176016 (2006h:46055)

[13] M. Neal, É. Ricard, and B. Russo, Classification of contractively complemented Hilbertian operator spaces, J. Funct. Anal. 237 (2006), 589-616. MR.2230351 (2007d:46050)

[14] P. W. Ng and N. Ozawa, A characterization of completely 1-complemented subspaces of noncommutative L $L_{1}$-spaces, Pacific J. Math. 205 (2002), 171-195. MR.1921083 (2003k:46094)

[15] S. Neuwirth, Cycles and 1-unconditional matrices, Proc. London Math. Soc. 93 (2006), 761790. MR2266966 (2008b:47034)

[16] V. Paulsen, Completely bounded maps and operator algebras, Cambridge Studies in Advanced Math. 78, Cambridge University Press, Cambridge, 2002. MR.1976867(2004c:46118)

[17] G. Pisier, Non-commutative vector valued $L_{p}$-spaces and completely $p$-summing maps, Soc. Math. France, Astérisque 247, 1998. MR1648908 (2000a:46108)

[18] G. Pisier, Introduction to operator space theory, London Math. Soc. Lecture Note Series 294, Cambridge University Press, 2003. MR.2006539 (2004k:46097)

[19] G. Pisier and Q. Xu, Non-commutative $L^{p}$-spaces, pp. 1459-1517 in "Handbook of the Geometry of Banach Spaces", Vol. II, edited by W.B. Johnson and J. Lindenstrauss, Elsevier, 2003. MR:1999201 (2004i:46095)

[20] R. Plymen and D. Robinson, Spinors on Hilbert spaces, Cambridge Tracts in Math. 114, Cambridge University Press, 1994.

[21] J. Tomiyama, On the projection of norm one in $W^{*}$-algebras, III, Tohoku Math. J. 11 (1959), 125-129. MR.0107825 (21:6547)

Laboratoire de Mathématiques, Université de Franche-Comté, 25030 Besançon Cedex, France

E-mail address: clemerdy@univ-fcomte.fr

Laboratoire de Mathématiques, Université de Franche-Comté, 25030 Besançon Cedex, France

E-mail address: eric.ricard@univ-fcomte.fr

Laboratoire de Mathématiques, Université de Franche-Comté, 25030 Besançon Cedex, France

E-mail address: jean.roydor@univ-fcomte.fr 